\title{
Synthesis and evaluation of anthranilamide-based derivatives as FXa inhibitors
}

\author{
Changjiang Huang ${ }^{1,2}$, Wenzhi Wang ${ }^{3}$, Yao Li², Shijun Zhang ${ }^{2}$, Fancui Meng², Weiren \\ $\mathrm{Xu}^{2}$, Jing Yuan ${ }^{2}$, Ligong Chen ${ }^{1,4,5}$ \\ ${ }^{1}$ School of Chemical Engineering and Technology, Tianjin University, Tianjin, China \\ ${ }^{2}$ Tianjin Key Laboratory of Molecular Design and Drug Discovery, Tianjin Institute of Pharmaceutical Research, Tianjin, China \\ ${ }^{3}$ School of Pharmaceutical Engineering, Shenyang Pharmaceutical University, Shenyang, China \\ ${ }^{4}$ Collaborative Innovation Center of Chemical Science and Engineering, Tianjin, China \\ ${ }^{5}$ Tianjin Engineering Research Center of Functional Fine Chemicals, Tianjin, China \\ Correspondence to: Jing Yuan, email: yuanj@tjipr.com \\ Ligong Chen, email: Igchen@tju.edu.cn
}

Keywords: thrombosis, thromboembolic diseases, anticoagulants, factor Xa inhibitors, thrombin and docking simulation

Received: November 23, 2016

Accepted: March 10, 2017

Published: March 21, 2017

Copyright: Huang et al. This is an open-access article distributed under the terms of the Creative Commons Attribution License (CC-BY), which permits unrestricted use, distribution, and reproduction in any medium, provided the original author and source are credited.

\section{ABSTRACT}

Factor Xa ( FXa) plays a significant role in the blood coagulation cascade and is a promising target for anticoagulation drugs. Three oral FXa inhibitors have been approved by FDA for treating thrombotic diseases. In this study, 43 novel compounds were synthesized anthranilamide-based FXa inhibitors aiming to ameliorate the toxicity of traditional FXa inhibitors in clinic. The data indicated that the compounds $6 a, 6 a-$ b, 6a-e, 6k, 6k-a and 6k-b showed remarkable FXa inhibitory activity and excellent selectivity over thrombin in vitro. Selected compounds also exhibited anticoagulant activities in vitro consequently and were potent novel anti-coagulators in further.

\section{INTRODUCTION}

The coagulation and anticoagulation process was considered as a dynamic equilibrium process, the breakage of this balance was capable to induce thrombosis [1]. Warfarin was employed as oral anticoagulant for decades [2] however the clinical utility of warfarin was limited by its narrow therapeutic index, dietary restrictions, slow onset of action and the need for regular monitoring $[3,4]$. In order to overcome this clinical obstacle of warfarin, researchers focused on developing novel synthetic molecules to inhibit specific enzymes such as protease factor $\mathrm{Xa}(\mathrm{FXa})$ [5]. FXa is a junction of the intrinsic and extrinsic pathways, and it was convinced to be a key component in the coagulation factor activation and thrombosis formation [6]. Up to date, three oral direct FXa inhibitors such as Rivaroxaban have been approved to treat the venous thrombosis in clinics and several other candidates were in the variant stages of clinical studies or biological testing (the structures of three approved drugs were in Figure 1) [7]. However, these three medicines still possess specific obstacles in clinical utility, such as risk of bleeding (including intracranial bleeding, gastrointestinal bleeding, epistaxis and other fatal bleeds), no antidote and higher incidence of thromboembolic events after ceasing treatment [8]. Therefore, the development of novel antithrombotic drugs is still attracted many attention for unmet clinical demand.

According to structural analysis, the carboxamide group at both rivaroxaban and betrixaban was considered to play a crucial role for connecting the scaffold, P1 and P4. Betrixaban interacts with residues Gly218 and Gly216 in FXa by two hydrogen bonds [7]. All these structural information supported a hypothesis that the carboxamide group might be crucial component for the docking of FXa inhibitors with human FXa protein. In this study, we synthesized 43 novel compounds in which carboxamide group was involved.

Additionally, the clinical pharmacokinetics investigations of rivaroxaban revealed that the metabolic pathway of rivaroxaban is to hydrolyze the amide bond and then eliminate via renal and biliary/fecal routes rapidly [9]. The metabolic characterizations of rivaroxaban suggested less toxicity by reversing the order of chemical group in betrixaban which was presumed to result in totally different metabolic pathways in humans even. 
In brief, we designed and evaluated novel synthesized compounds as inhibitors of FXa and their structural information upon computational simulation approaches.

\section{RESULTS AND DISCUSSION}

\section{Synthesis}

The compounds listed in Tables 1 and 2 are prepared as shown in Figure 2. The compound 3 was synthesized from the anilines 2 and o-nitrobenzoyl chloride 1, Then the anilines 4 was prepared by reduction reaction. Compound 6 was accessed by treatment of compound 4 with compound 5. The compound's Activity for inhibit FXa was reported as IC50 and we also reported the Ki of several more potent compounds.

\section{In Vitro inhibition activity studies on FXa}

We extensively investigated different P1 group. Important SAR findings are summarized in Table 1. Compounds 6a-6o that contained same scaffold and P4 group were used for choosing potent $\mathrm{P} 1$ group. Compounds $6 \mathrm{a}, 6 \mathrm{~h}, 6 \mathrm{k}$ and 61 exhibited a promising inhibitory activity against $\mathrm{FXa}$ with $\mathrm{IC}_{50}$ values of 28.7, 277.5, 23.8 and $181.5 \mathrm{nM}$. The P1 group of $6 \mathrm{a}, 6 \mathrm{~h}, 6 \mathrm{k}$ and 61 were chosen for structural modification of next stage and provided an opportunity to improve potency.

Then, the SAR of substituent on benzene ring and different P4 group were examined (Table 2). Unfortunately, the 2-chloropyridine analogs (6h-a - 6h-g) and 4-methoxybenzene analogs $(61-a-61-g)$ still did not exhibited good activity. However, 5-bromothiophene analogs and 2, 4-dichlorobenzene analogs both showed excellent activity. In particular compounds $6 \mathrm{a}-\mathrm{b}$ and $6 \mathrm{k}-\mathrm{b}$ showed some validity with the $\mathrm{IC}_{50}$ value of 18.8 and $35.5 \mathrm{nM}$. From these results we can find that 3-methylsubstituted scaffold (6a-c, 6a-g, 6k-c and 6k-g) was not suitable, the related compounds displayed poor $\mathrm{IC}_{50}$ values at a micromole level no matter what kind of $R_{2}$ and $R_{3}$. Compounds with 5-electron donating group-substituted scaffold exhibited almost 10 fold better than 5-electron withdrawing group substituted scaffold. The $\mathrm{IC}_{50}$ value of compounds with non-substituted scaffold was almost at the same level with electron donating group scaffold.

\section{Thrombin selectivity comparison and assay of prothrombin time (PT)}

Table 3 shows the thrombin selectivity comparison and the extension of the prothrombin time (PT) of $6 \mathrm{a}$, $6 \mathrm{a}-\mathrm{b}, 6 \mathrm{a}-\mathrm{e}, 6 \mathrm{k}, 6 \mathrm{k}-\mathrm{a}$ and $6 \mathrm{k}-\mathrm{b}$. The IC50 of rivaroxaban against thrombin is $6.9 \mu \mathrm{M}$ [10], our compounds were also highly selective, $\mathrm{IC}_{50}$ were all far higher than $10 \mu \mathrm{M}$. The assay of PT also showed that our compound possessed improvement on anticoagulant selectivity.

\section{Docking simulation studies}

In order to analyze the detailed interactions between compounds 6a, 6a-b, 6a-e, 6k, 6k-a and 6k-b to FXa, the computational docking simulation studies were performed. As showed in Figure 3, There are residue Gly216 and Gly218 in S1 pocket of FXa, and some hydrogen bonds were taked shape betweed conpound and these amino-acid residue. the unit of pyridone or morpholino are access

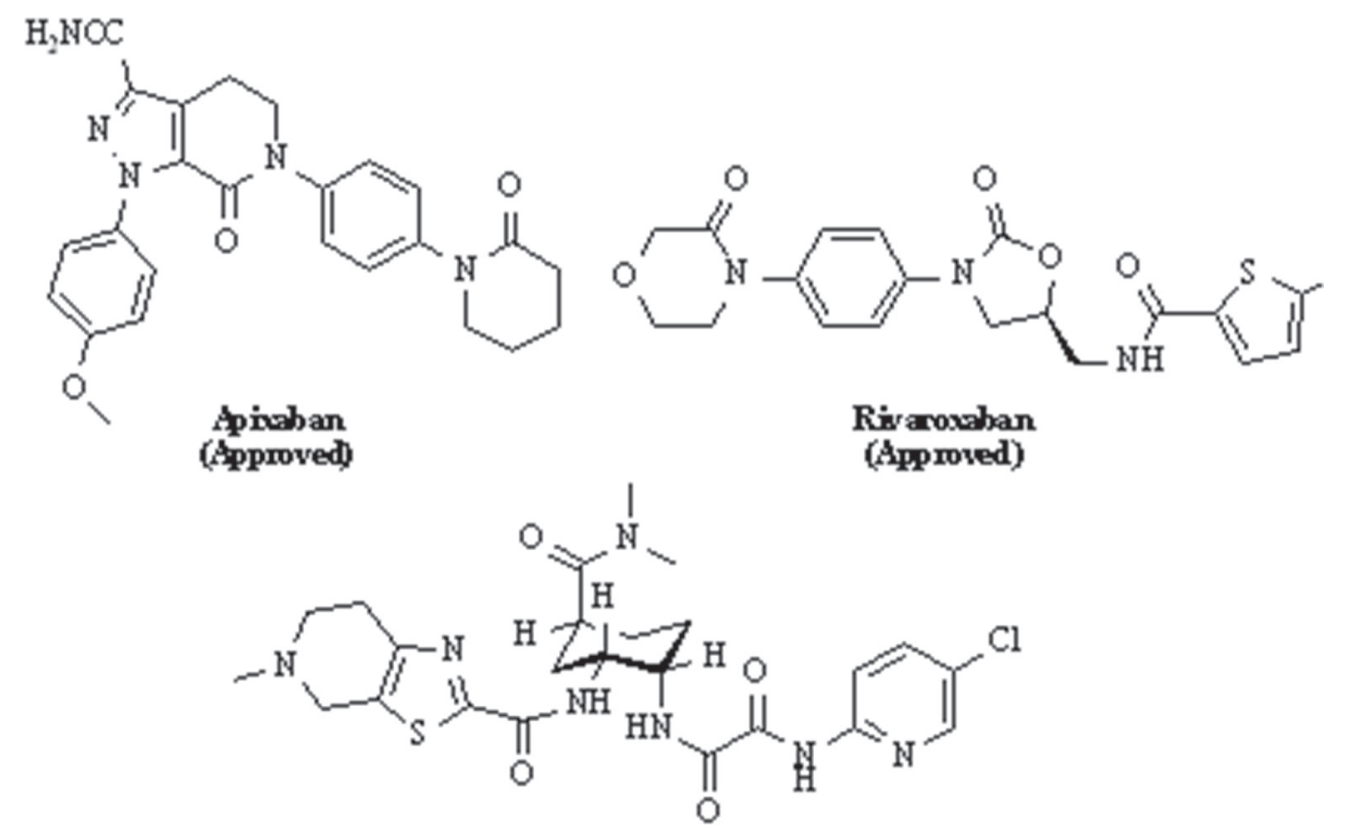

Edacaban

Figure 1: Oral direct FXa inhibitors. 
Table 1: Representative SAR for the P1 variants

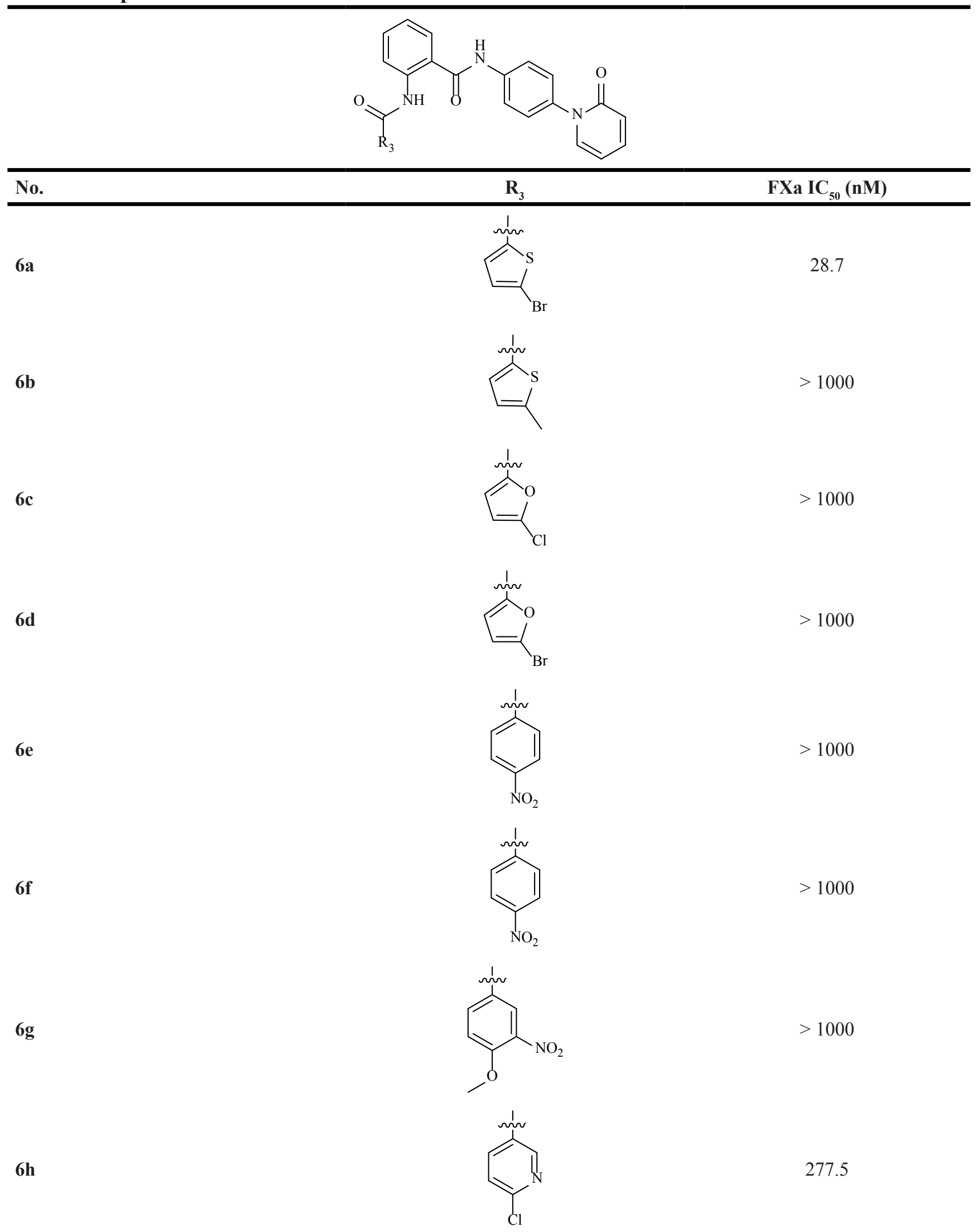


<smiles>Fc1cc(I)cc(I)c1F</smiles>

6k<smiles>Cc1ccc(Cl)cc1Cl</smiles>

61<smiles>COc1ccc(C(C)(C)C)cc1</smiles><smiles>COc1ccc([N+]([N+])([O-])[O-])cc1OC</smiles><smiles>COc1cc(I)cc(OC)c1OC</smiles>

the S4 pocket of FXa, which was composed of Tyr99, Phe174 and Trp215. The p-p conjugation was formed between the chloro- or bromo- substituent and S1 pocket through amino-acid residue Tyr228. The mode of action was similar with rivaroxaban [7].

\section{MATERIALS AND METHODS}

Reagents and solvents were obtained from commercial suppliers and used as received without further purification. All reactions were monitored by thin layer chromatography. ${ }^{1} \mathrm{H}$ NMR spectra (400 MHz) were recorded for DMSO- $d 6$ solutions on an AV400
NMR (Bruker, Billerica, MA, USA), MS were measured on a Finnigan LCQ Mass (Thermo Fisher Scientific, Cambridge, MA, USA), HRMS were measured on a miorOTOF-QII instrument (Bruker Daltonics, Billerica, MA, USA) and melting points (uncorrected) were determined on a YRT-3 Melting Point Tester (Precision Instrument of Tianjin University, Tianjin, China).

\section{Synthesis of 3 (3a-3h)}

To a stirred solution of compound 2 (4.8 mmol) (2a-2b), $\mathrm{K}_{2} \mathrm{CO}_{3}(0.80 \mathrm{~g}, 5.8 \mathrm{mmol})$ and DMAP (0.05 g, $0.4 \mathrm{mmol})$ in THF $(20 \mathrm{~mL})$, solution of compound 1 


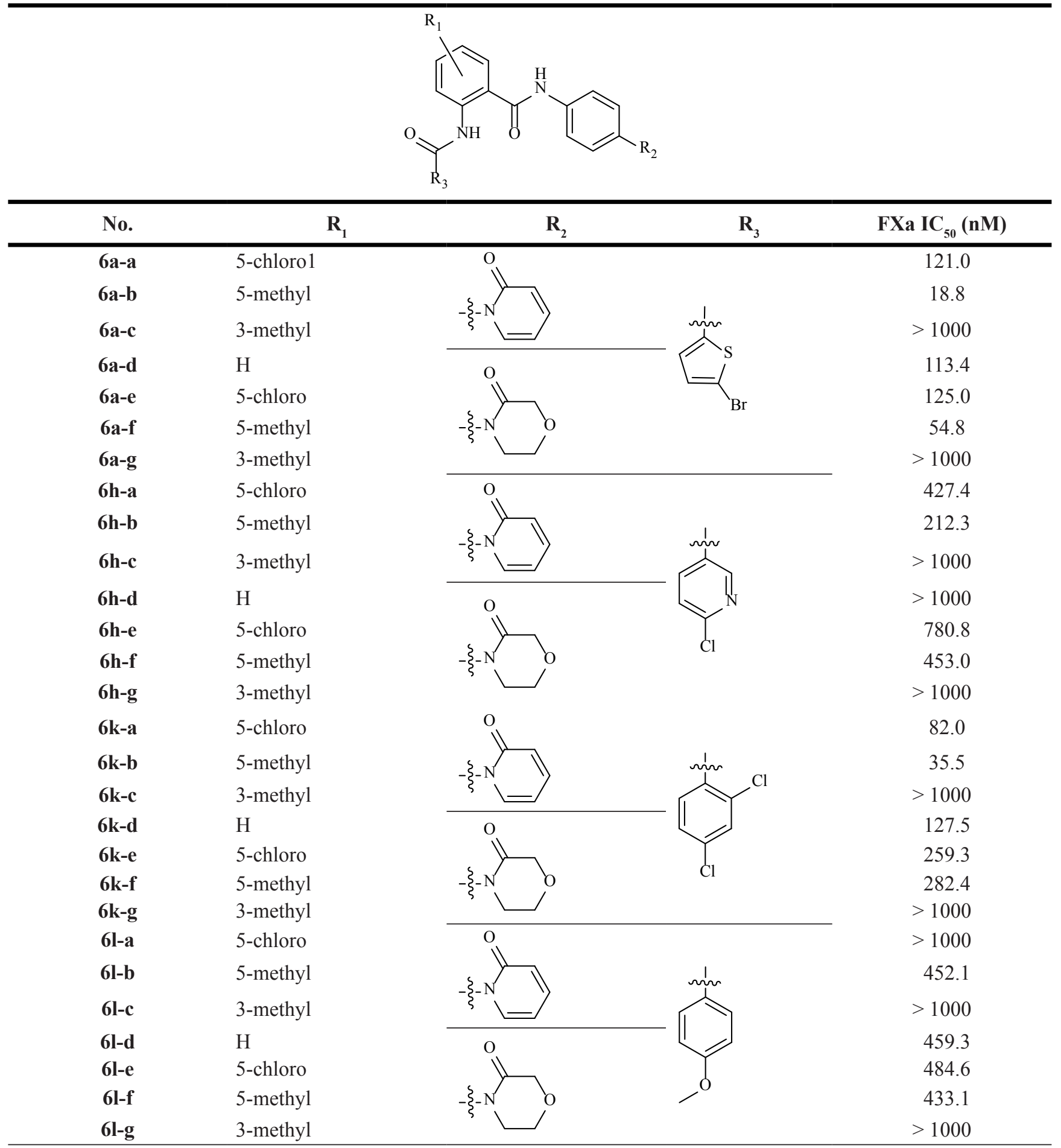

(6.24 mmol) (1a-1d) in THF (5 mL) was added at room temperature and the mixture was refluxed for $2 \mathrm{~h}$. The reaction mixture was cooled down to room temperature and concentrated under reduced pressure. Then water (100 $\mathrm{mL}$ ) was added to the mixture and stirred for $10 \mathrm{~min}$ at room temperature. The resulting precipitate was collected by filtration. The reaction was monitored by TLC with EA.

\section{Synthesis of 4 (4a-4h)}

To a $250 \mathrm{~mL}$ round bottom flask, compound 3 (3.9 mmol) (3a-3h), zinc powder (2.05 g, $31.2 \mathrm{mmol})$, $\mathrm{NH}_{4} \mathrm{Cl}(2.11 \mathrm{~g}, 39 \mathrm{mmol})$, methanol $(30 \mathrm{~mL})$, THF $(30 \mathrm{~mL})$ and water $(15 \mathrm{~mL})$ were added. The mixture stirred at $40^{\circ} \mathrm{C}$ for $2 \mathrm{~h}$. The reaction mixture was filtered, washed 
Table 3: Anticoagulant activity of 6a, 6a-b, 6a-e, 6k, 6k-a and 6k-b

\begin{tabular}{lcc}
\hline No. & FXa $\mathbf{K i}(\mathbf{n M})$ & $\mathbf{2} \times \mathbf{P T}(\boldsymbol{\mu M})($ Human $)$ \\
\hline $\mathbf{6 a}$ & 20.5 & 8.4 \\
$\mathbf{6 a - b}$ & 13.4 & 4.2 \\
$\mathbf{6 a - e}$ & 39.1 & 3.8 \\
$\mathbf{6 k}$ & 17.0 & 16.4 \\
$\mathbf{6 k - a}$ & 58.6 & 16.9 \\
$\mathbf{6 k - b}$ & 25.4 & 11.3 \\
rivaroxaban & 0.7 & 0.2 \\
\hline
\end{tabular}

with DMF and the filtrate was concentrated under reduced pressure. Then water $(200 \mathrm{~mL})$ was added to the mixture and stirred for $0.5 \mathrm{~h}$. The residue was filtered and washed with water to yield the title compound 4 (4a-4h). The reaction was monitored by TLC with EA.

\section{Synthesis of 6 (6a-6o, 6a-a-6a-g, 6h-a-6h-g, 6h-a-6h-g, 6l-a-6l-g)}

To a stirred solution of compound $4(0.98 \mathrm{mmol})$ (4a-4h), $\mathrm{K}_{2} \mathrm{CO}_{3}(0.16 \mathrm{~g}, 1.18 \mathrm{mmol})$ and $\operatorname{DMAP}(0.01 \mathrm{~g}$, $0.08 \mathrm{mmol})$ in THF $(10 \mathrm{~mL})$, solution of compound 5 $(1.27 \mathrm{mmol})(5 \mathrm{a}-5 \mathrm{~h})$ in THF $(5 \mathrm{~mL})$ was added at room temperature and the mixture was refluxed for $4 \mathrm{~h}$. The reaction mixture was cool down to room temperature and water $(30 \mathrm{~mL})$ was added. The resulting precipitate was collected by filtration. The authentic sample was prepared by recrystallization from $\mathrm{DMF} / \mathrm{MeOH}$. The reaction was monitored by TLC with EA.

\section{Spectral date}

2-Nitro-N-(4-(2-oxopyridin-1(2H)-yl)phenyl)benzamide (3a)

White solid product (1.46 g, 90\%). MS: $[\mathrm{M}+\mathrm{H}]^{+}$ 336.22. ${ }^{1} \mathrm{H}$ NMR: $\delta$ ppm $6.30(\mathrm{t}, J=6.4 \mathrm{~Hz}, 1 \mathrm{H}), 6.47$ (d, $J=9.2 \mathrm{~Hz}, 1 \mathrm{H}), 7.39$ (d, $J=8.8 \mathrm{~Hz}, 2 \mathrm{H}), 7.49$ (t, $J=$ $4.4 \mathrm{~Hz}, 1 \mathrm{H}), 7.63(\mathrm{~d}, J=6.8 \mathrm{~Hz}, 1 \mathrm{H}), 7.76-7.80(\mathrm{~m}, 4 \mathrm{H})$, $7.89(\mathrm{t}, J=7.6 \mathrm{~Hz}, 1 \mathrm{H}), 8.17(\mathrm{~d}, J=8.0 \mathrm{~Hz}, 1 \mathrm{H}), 10.84$ (s, NH).

5-Chloro-2-nitro-N-(4-(2-oxopyridin-1(2H)-yl)phenyl)benzamide (3b)

White solid product (1.64 g, 92\%). MS: $[\mathrm{M}+\mathrm{H}]^{+}$ 370.05. ${ }^{1} \mathrm{H}$ NMR: $\delta$ ppm $6.31(\mathrm{t}, J=6.8 \mathrm{~Hz}, 1 \mathrm{H}), 6.47$ $(\mathrm{d}, J=9.2 \mathrm{~Hz}, 1 \mathrm{H}), 7.40(\mathrm{~d}, J=8.4 \mathrm{~Hz}, 2 \mathrm{H}), 7.50$ (t, $J=2.4 \mathrm{~Hz}, 1 \mathrm{H}), 7.63(\mathrm{~d}, J=8.8 \mathrm{~Hz}, 1 \mathrm{H}), 7.76$ (d, $J=6.8 \mathrm{~Hz}, 2 \mathrm{H}), 7.86(\mathrm{~d}, J=6.4 \mathrm{~Hz}, 1 \mathrm{H}), 8.00(\mathrm{~s}, 1 \mathrm{H})$, $8.20(\mathrm{~d}, J=8.8 \mathrm{~Hz}, 1 \mathrm{H}), 10.88(\mathrm{~s}, \mathrm{NH})$.<smiles>[R]c1ccc(C(=O)Cl)c([N+](=O)[O-])c1</smiles>

1<smiles>[R]c1ccc(N)cc1</smiles>

2<smiles>[R1]C=CC(C(=O)Nc1ccc([R])cc1)=C(C=C)[N+](=O)[O-]</smiles>

3<smiles>[R]C=CC(C(N)=O)=C(N)/C=C\C(=O)Nc1ccc([R])cc1</smiles>

4

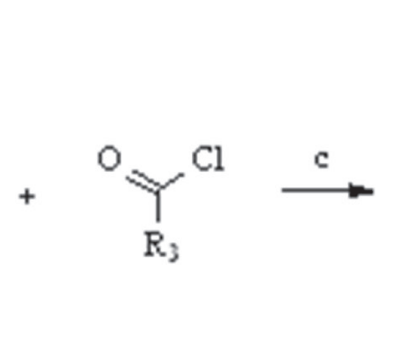

5<smiles>[R]C=CC(NC([R])=O)=C(/C=C\C)C(=O)Nc1ccc([R])cc1</smiles>

6

Figure 2: Synthesis of Compound 6. Reagent and conditions: (A) THF, $\mathrm{K}_{2} \mathrm{CO}_{3}$, DMAP, reflux, $2 \mathrm{~h}$; (B) $\mathrm{Zn}, \mathrm{NH}_{4} \mathrm{Cl}, \mathrm{H}_{2} \mathrm{O}, \mathrm{THF}, \mathrm{MeOH}$, $40^{\circ} \mathrm{C}, 2 \mathrm{~h} ;(\mathbf{C}) \mathrm{THF}, \mathrm{K}_{2} \mathrm{CO}_{3}$, DMAP, reflux, $4 \mathrm{~h}$. 
5-Methyl-2-nitro-N-(4-(2-oxopyridin-1(2H)-yl) phenyl) benzamide (3c): White solid product (1.50 g, 89\%). MS: $[\mathrm{M}+\mathrm{H}]^{+} 350.42 .{ }^{1} \mathrm{H}$ NMR: $\delta$ ppm 2.43 (s, 3H), $6.31(\mathrm{t}, J=6.4 \mathrm{~Hz}, 1 \mathrm{H}), 6.47$ (d, $J=9.2 \mathrm{~Hz}, 1 \mathrm{H}), 7.38$ $(\mathrm{d}, J=8.8 \mathrm{~Hz}, 2 \mathrm{H}), 7.50(\mathrm{t}, J=4.8 \mathrm{~Hz}, 1 \mathrm{H}), 7.55-7.64$ (m, 3H), $7.76(\mathrm{~d}, J=9.2 \mathrm{~Hz}, 2 \mathrm{H}), 8.08(\mathrm{~d}, J=8.4 \mathrm{~Hz}, 1 \mathrm{H})$, $10.78(\mathrm{~s}, \mathrm{NH})$.

3-Methyl-2-nitro-N-(4-(2-oxopyridin-1(2H)-yl)phenyl) benzamide (3d)

White solid product $(1.54 \mathrm{~g}, 91 \%)$. MS: $[\mathrm{M}+\mathrm{H}]^{+}$ 350.1. ${ }^{1} \mathrm{HNMR}: \delta \mathrm{ppm} 2.36\left(\mathrm{~s}, \mathrm{CH}_{3}\right), 6.30(\mathrm{t}, J=6.4 \mathrm{~Hz}, 1 \mathrm{H})$, $6.46(\mathrm{~d}, J=9.2 \mathrm{~Hz}, 1 \mathrm{H}), 7.38-7.40(\mathrm{~m}, 2 \mathrm{H}), 7.49(\mathrm{t}$, $J=8.8 \mathrm{~Hz}, 1 \mathrm{H}), 7.62(\mathrm{~d}, J=8.8 \mathrm{~Hz}, 1 \mathrm{H}), 7.64-7.67$
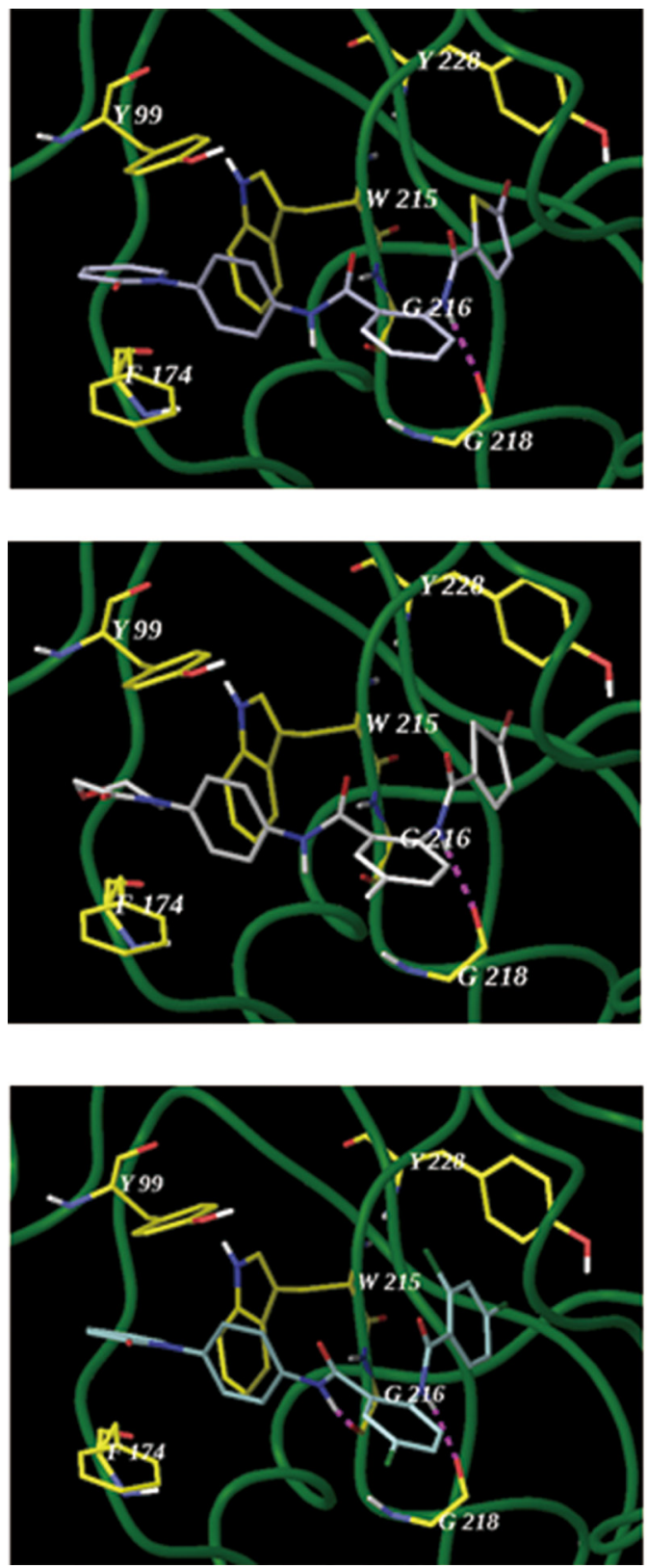

(m, 2H), 7.69-7.73 (m, 1H),7.77 (d, J=8.8 Hz, 2H), 10.87 (s, NH).

2-Nitro-N-(4-(3-oxomorpholino)phenyl)benzamide (3e)

White solid product (1.54 g, $93 \%)$. MS: $[\mathrm{M}+\mathrm{H}]^{+}$ 342.09. ${ }^{1} \mathrm{H}-\mathrm{NMR}: \delta$ ppm $3.71\left(\mathrm{t}, J=4.8 \mathrm{~Hz}, \mathrm{CH}_{2}\right), 3.97$ $\left(\mathrm{t}, J=4.8 \mathrm{~Hz}, \mathrm{CH}_{2}\right), 4.19(\mathrm{~s}, 2 \mathrm{H}), 7.37(\mathrm{~d}, J=8.8 \mathrm{~Hz}, 2 \mathrm{H})$, $7.67(\mathrm{~d}, J=8.8 \mathrm{~Hz}, 2 \mathrm{H}), 7.74-7.78(\mathrm{~m}, 2 \mathrm{H}), 7.87$ $(\mathrm{t}, J=7.6 \mathrm{~Hz}, 1 \mathrm{H}), 8.15(\mathrm{~d}, J=8.0 \mathrm{~Hz}, 1 \mathrm{H}), 10.72(\mathrm{~s}, \mathrm{NH})$.

5-Chloro-2-nitro-N-(4-(3-oxomorpholino)phenyl)benzamide (3f)

White solid product $(1.67 \mathrm{~g}, 94 \%)$. MS: $[\mathrm{M}+\mathrm{H}]^{+}$ 376.05. ${ }^{1} \mathrm{H}$ NMR: $\delta$ ppm $3.72(\mathrm{t}, J=10.0 \mathrm{~Hz}, 2 \mathrm{H}), 3.97$
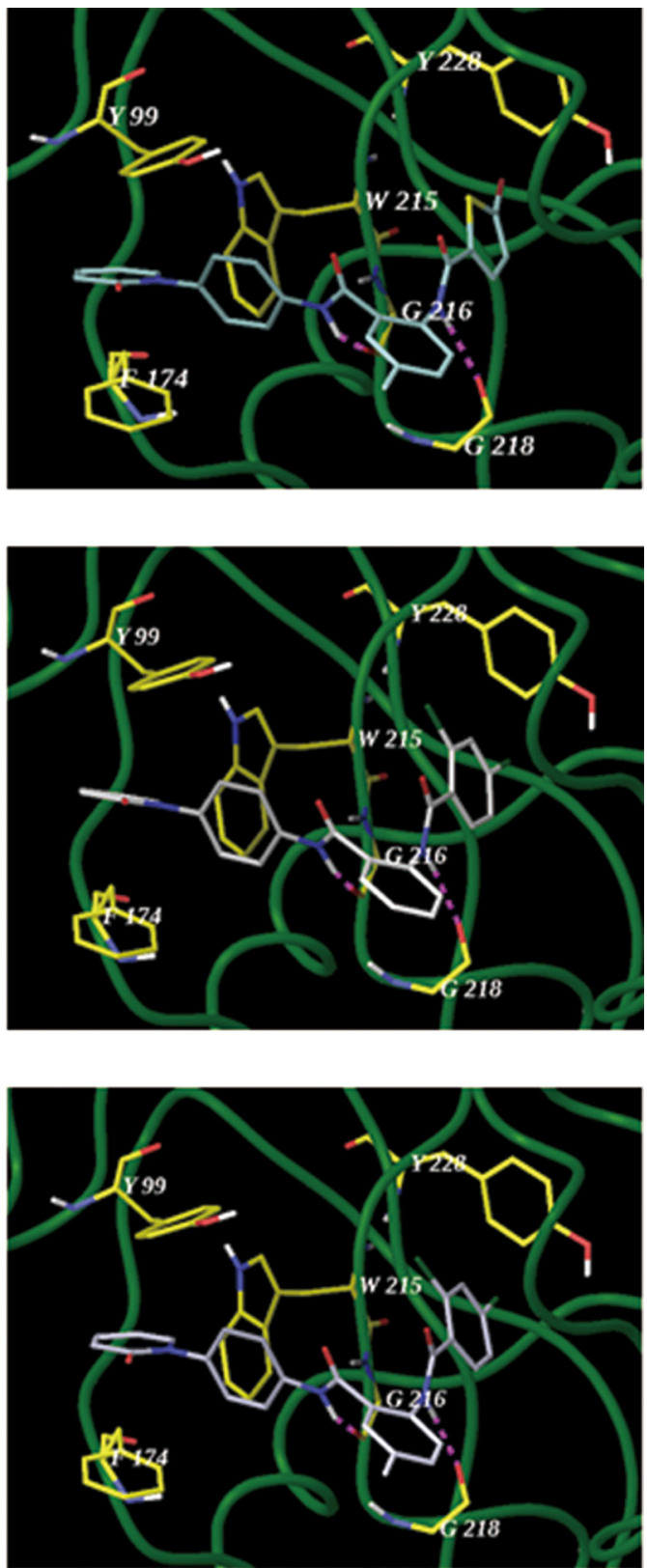

Figure 3: The interactions of compounds 6a (1st panel, PDB code 2xbv), 6a-b (2nd panel), 6a-e (3rd panel), 6k (4th panel), 6k-a (5th panel), 6k-b (6th panel) with the active site of FXa. 
(t, $J=10.0 \mathrm{~Hz}, 2 \mathrm{H}), 4.19(\mathrm{~s}, 2 \mathrm{H}), 7.38(\mathrm{~d}, J=8.4 \mathrm{~Hz}, 2 \mathrm{H})$, 7.66 (d, $J=8.8 \mathrm{~Hz}, 2 \mathrm{H}), 7.84$ (d, $J=8.8 \mathrm{~Hz}, 2 \mathrm{H}), 7.94$ (s, 1H), $8.18(\mathrm{~d}, J=8.8 \mathrm{~Hz}, 2 \mathrm{H}), 10.77$ (s, NH).

\section{5-Methyl-2-nitro-N-(4-(3-oxomorpholino)phenyl)benz- amide (3g)}

White solid product (1.55 g, 90\%). MS: $[\mathrm{M}+\mathrm{H}]^{+}$ 355.99. ${ }^{1} \mathrm{H}$ NMR: $\delta$ ppm $2.47\left(\mathrm{~s}, \mathrm{CH}_{3}\right), 3.71(\mathrm{t}, J=4.8$ $\left.\mathrm{Hz}, \mathrm{CH}_{2}\right), 3.97\left(\mathrm{t}, J=4.8 \mathrm{~Hz}, \mathrm{CH}_{2}\right), 4.19\left(\mathrm{~s}, \mathrm{CH}_{2}\right), 7.37$ $(\mathrm{d}, J=8.8 \mathrm{~Hz}, 2 \mathrm{H}), 7.55(\mathrm{~d}, J=8.8 \mathrm{~Hz}, 2 \mathrm{H}), 7.57(\mathrm{~s}, 1 \mathrm{H})$, $7.67(\mathrm{~d}, J=8.8 \mathrm{~Hz}, 2 \mathrm{H}), 8.07$ (d, $J=8.4 \mathrm{~Hz}, 1 \mathrm{H}), 10.66$ $(\mathrm{s}, \mathrm{NH})$.

\section{3-Methyl-2-nitro-N-(4-(3-oxomorpholino)phenyl)benz- amide (3h)}

White solid product $(1.53 \mathrm{~g}, 89 \%)$. MS: $[\mathrm{M}+\mathrm{H}]^{+}$ 356.19. ${ }^{~} \mathrm{H}$ NMR: $\delta$ ppm 2.36 (s, $\left.\mathrm{CH}_{3}\right), 3.72$ (t, $J=4.8 \mathrm{~Hz}$, $\left.\mathrm{CH}_{2}\right), 3.98\left(\mathrm{t}, J=4.8 \mathrm{~Hz}, \mathrm{CH}_{2}\right), 4.20\left(\mathrm{~s}, \mathrm{CH}_{2}\right), 7.38$ (d, $J=8.8 \mathrm{~Hz}, 2 \mathrm{H}), 7.65-7.24(\mathrm{~m}, 5 \mathrm{H}), 10.76(\mathrm{~s}, \mathrm{NH})$.

\section{2-Amino-N-(4-(2-oxopyridin-1(2H)-yl)phenyl)benzam- ide (4a)}

White solid product (1.13 g, 89\%).MS: $[\mathrm{M}+\mathrm{H}]^{+}$ 306.10. ${ }^{1} \mathrm{H}$ NMR: $\delta$ ppm $6.30(\mathrm{t}, J=6.4 \mathrm{~Hz}, 1 \mathrm{H}), 6.34$ $\left(\mathrm{s}, \mathrm{NH}_{2}\right), 6.46(\mathrm{~d}, J=8.8 \mathrm{~Hz}, 1 \mathrm{H}), 6.60(\mathrm{t}, J=7.2 \mathrm{~Hz}$, $1 \mathrm{H}), 6.76(\mathrm{~d}, J=8.4 \mathrm{~Hz}, 1 \mathrm{H}), 7.21(\mathrm{t}, J=7.2 \mathrm{~Hz}, 1 \mathrm{H})$, $7.33-7.36(\mathrm{~m}, 2 \mathrm{H}), 7.49(\mathrm{t}, J=8.8 \mathrm{~Hz}, 1 \mathrm{H}), 7.61-7.65$ $(\mathrm{m}, 2 \mathrm{H}), 7.83(\mathrm{~d}, J=8.8 \mathrm{~Hz}, 2 \mathrm{H}), 10.14(\mathrm{~s}, \mathrm{NH})$.

\section{2-Amino-5-chloro-N-(4-(2-oxopyridin-1(2H)-yl)phenyl)} benzamide (4b)

Yellow solid product $(1.29 \mathrm{~g}, 91 \%)$. MS: $[\mathrm{M}+\mathrm{H}]^{+}$ 340.10. ${ }^{1} \mathrm{H}$ NMR: $\delta$ ppm $6.30(\mathrm{t}, J=5.2 \mathrm{~Hz}, 1 \mathrm{H}), 6.47$ (d, $J=10.4 \mathrm{~Hz}, 1 \mathrm{H}), 6.48\left(\mathrm{~s}, \mathrm{NH}_{2}\right), 6.78(\mathrm{~d}, J=9.2 \mathrm{~Hz}, 1 \mathrm{H})$, $7.24(\mathrm{~d}, J=6.8 \mathrm{~Hz}, 1 \mathrm{H}), 7.35$ (d, $J=8.8 \mathrm{~Hz}, 2 \mathrm{H}), 7.49$ (t, $J=7.2 \mathrm{~Hz}, 1 \mathrm{H}), 7.63$ (d, $J=8.4 \mathrm{~Hz}, 1 \mathrm{H}), 7.70(\mathrm{~s}, 1 \mathrm{H})$, $7.80(\mathrm{~d}, J=8.8 \mathrm{~Hz}, 2 \mathrm{H}), 10.24$ (s, NH).

\section{2-Amino-5-methyl-N-(4-(2-oxopyridin-1(2H)-yl) phenyl) benzamide (4c)}

Yellow solid product (1.23 g, 92\%). MS: $[\mathrm{M}+\mathrm{H}]^{+}$ 320.04. ${ }^{1} \mathrm{H}$ NMR: $\delta$ ppm 2.22 (s, $\left.\mathrm{CH}_{3}\right), 6.11\left(\mathrm{~s}, \mathrm{NH}_{2}\right), 6.30$ $(\mathrm{t}, J=6.4 \mathrm{~Hz}, 1 \mathrm{H}), 6.46(\mathrm{~d}, J=8.8 \mathrm{~Hz}, 1 \mathrm{H}), 6.68(\mathrm{~d}, J=8.4 \mathrm{~Hz}$, 1H), 7.04 (d, $J=8.0 \mathrm{~Hz}, 1 \mathrm{H}), 7.34$ (d, $J=8.8 \mathrm{~Hz}, 2 \mathrm{H}), 7.44$ (s, 1H), 7.49 (t, $J=8.8 \mathrm{~Hz}, 1 \mathrm{H}), 7.63(\mathrm{~d}, J=8.8 \mathrm{~Hz}, 1 \mathrm{H})$, 7.81 (d, $J=8.8 \mathrm{~Hz}, 2 \mathrm{H}), 10.11(\mathrm{~s}, \mathrm{NH})$.

\section{2-Amino-3-methyl-N-(4-(2-oxopyridin-1(2H)-yl) phenyl) benzamide (4d)}

White solid product $(1.20 \mathrm{~g}, 90 \%)$. MS: $[\mathrm{M}+\mathrm{H}]^{+}$ 320.04. ${ }^{1} \mathrm{H}$ NMR: $\delta$ ppm $2.12\left(\mathrm{~s}, \mathrm{CH}_{3}\right), 6.13\left(\mathrm{~s}, \mathrm{NH}_{2}\right)$, $6.30(\mathrm{t}, J=6.8 \mathrm{~Hz}, 1 \mathrm{H}), 6.46(\mathrm{~d}, J=9.2 \mathrm{~Hz}, 1 \mathrm{H}), 6.58$ $(\mathrm{t}, J=7.2 \mathrm{~Hz}, 1 \mathrm{H}), 7.15(\mathrm{~d}, J=7.2 \mathrm{~Hz}, 1 \mathrm{H}), 7.34(\mathrm{~d}, J=8.8 \mathrm{~Hz}$, 2H), 7.47-7.54 (m, 2H), $7.62(\mathrm{~d}, J=8.4 \mathrm{~Hz}, 1 \mathrm{H}), 7.82$ (d, $J=8.8 \mathrm{~Hz}, 2 \mathrm{H}), 10.17$ (s, NH).
2-Amino-N-(4-(3-oxomorpholino)phenyl)benzamide (4e)

White solid product $(1.21 \mathrm{~g}, 93 \%)$. MS: $[\mathrm{M}+\mathrm{H}]^{+}$ 312.04. ${ }^{1} \mathrm{H}$ NMR: $\delta$ ppm $3.71\left(\mathrm{t}, J=4.8 \mathrm{~Hz}, \mathrm{CH}_{2}\right), 3.96$ (t, $\left.J=4.8 \mathrm{~Hz}, \mathrm{CH}_{2}\right), 4.18\left(\mathrm{~s}, \mathrm{CH}_{2}\right), 6.31\left(\mathrm{~s}, \mathrm{NH}_{2}\right), 6.58$ (t, $J=7.2 \mathrm{~Hz}, 1 \mathrm{H}), 6.74(\mathrm{~d}, J=8.0 \mathrm{~Hz}, 1 \mathrm{H}), 7.13$ (t, $J=8.4 \mathrm{~Hz}, 1 \mathrm{H}), 7.33(\mathrm{~d}, J=7.2 \mathrm{~Hz}, 2 \mathrm{H}), 7.61$ (d, $J=8.8 \mathrm{~Hz}, 1 \mathrm{H}), 7.72$ (d, $J=8.8 \mathrm{~Hz}, 2 \mathrm{H}), 10.03$ (s, NH).

2-Amino-5-chloro-N-(4-(3-oxomorpholino)phenyl)benzamide (4f)

White solid product $(1.28 \mathrm{~g}, 89 \%)$. MS: $[\mathrm{M}+\mathrm{H}]^{+}$ 345.97. ${ }^{1} \mathrm{H}$ NMR: $\delta$ ppm $3.71\left(\mathrm{t}, J=4.8 \mathrm{~Hz}, \mathrm{CH}_{2}\right), 3.96$ (t, $\left.J=4.8 \mathrm{~Hz}, \mathrm{CH}_{2}\right), 4.19\left(\mathrm{~s}, \mathrm{CH}_{2}\right), 6.46\left(\mathrm{~s}, \mathrm{NH}_{2}\right), 6.78$ $(\mathrm{d}, J=8.8 \mathrm{~Hz}, 1 \mathrm{H}), 7.23(\mathrm{~d}, J=8.8 \mathrm{~Hz}, 2 \mathrm{H}), 7.34(\mathrm{~d}, J=8.8 \mathrm{~Hz}$, $2 \mathrm{H}), 7.68(\mathrm{~d}, J=7.6 \mathrm{~Hz}, 2 \mathrm{H}), 7.72(\mathrm{~s}, 1 \mathrm{H}), 10.14$ (s, NH).

\section{2-Amino-5-methyl-N-(4-(3-oxomorpholino)phenyl)be-} nzamide (4g)

Yellow solid product $(1.22 \mathrm{~g}, 90 \%)$. MS: $[\mathrm{M}+\mathrm{H}]^{+}$ 326.07. ${ }^{1} \mathrm{H}$ NMR: $\delta$ ppm $2.21\left(\mathrm{~s}, \mathrm{CH}_{3}\right), 3.71$ (t, $J=4.8 \mathrm{~Hz}$, $\left.\mathrm{CH}_{2}\right), 3.96\left(\mathrm{t}, J=4.8 \mathrm{~Hz}, \mathrm{CH}_{2}\right), 4.19\left(\mathrm{~s}, \mathrm{CH}_{2}\right), 6.09$ $\left(\mathrm{s}, \mathrm{NH}_{2}\right), 6.67(\mathrm{~d}, J=8.4 \mathrm{~Hz}, 1 \mathrm{H}), 7.03(\mathrm{~d}, J=8.0 \mathrm{~Hz}, 1 \mathrm{H})$, $7.33(\mathrm{~d}, J=8.8 \mathrm{~Hz}, 2 \mathrm{H}), 7.42(\mathrm{~s}, 1 \mathrm{H}), 7.72(\mathrm{~d}, J=8.8 \mathrm{~Hz}$, $2 \mathrm{H}), 10.01$ (s, NH).

2-Amino-3-methyl-N-(4-(3-oxomorpholino)phenyl)benzamide (4h)

White solid product (1.23 g, 91\%). MS: $[\mathrm{M}+\mathrm{H}]^{+}$ 326.03. ${ }^{1} \mathrm{H}$ NMR: $\delta$ ppm $2.11\left(\mathrm{~s}, \mathrm{CH}_{3}\right), 3.71$ (t, $J=4.8 \mathrm{~Hz}$, $\left.\mathrm{CH}_{2}\right), 3.96\left(\mathrm{t}, J=4.8 \mathrm{~Hz}, \mathrm{CH}_{2}\right), 4.19\left(\mathrm{~s}, \mathrm{CH}_{2}\right), 6.11$ $\left(\mathrm{s}, \mathrm{NH}_{2}\right), 6.56(\mathrm{t}, J=7.6 \mathrm{~Hz}, 1 \mathrm{H}), 7.13(\mathrm{~d}, J=6.8 \mathrm{~Hz}, 1 \mathrm{H})$, $7.33(\mathrm{~d}, J=8.8 \mathrm{~Hz}, 2 \mathrm{H}), 7.51(\mathrm{~d}, J=7.6 \mathrm{~Hz}, 1 \mathrm{H}), 7.72$ (d, $J=8.8 \mathrm{~Hz}, 2 \mathrm{H}), 10.06$ (s, NH).

5-bromo-N-(2-((4-(2-oxopyridin-1(2H)-yl)phenyl)carbamoyl)phenyl)thiophene-2-carboxamide (6a)

White solid product $(0.26 \mathrm{~g}, 56 \%)$, m.p. $>250^{\circ} \mathrm{C} .{ }^{1} \mathrm{H}$ NMR (400 MHz, DMSO): $\delta$ ppm $6.31(\mathrm{t}, J=6.8 \mathrm{~Hz}, 1 \mathrm{H})$, 6.47 (d, $J=8.8 \mathrm{~Hz}, 1 \mathrm{H}), 7.31-7.40$ (m, 4H), 7.47-7.52 (m, 1H), 7.58-7.64 (m, 3H), $7.81(\mathrm{~d}, J=8.4 \mathrm{~Hz}, 2 \mathrm{H}), 7.92$ (d, $J=7.6 \mathrm{~Hz}, 1 \mathrm{H}), 8.23(\mathrm{~d}, J=8.0 \mathrm{~Hz} 1 \mathrm{H}), 10.67(\mathrm{~s}, \mathrm{NH})$, 11.53 (s, NH). HRMS (ESI) calcd. for $\mathrm{C}_{23} \mathrm{H}_{16} \mathrm{BrN}_{3} \mathrm{O}_{3} \mathrm{~S}$ : $[\mathrm{M}+\mathrm{Na}]^{+} \mathrm{m} / \mathrm{z}$ : 515.9993, found: 515.9998 .

5-methyl-N-(2-((4-(2-oxopyridin-1(2H)-yl)phenyl)carbamoyl)phenyl)thiophene-2-carboxamide (6b)

Yellow solid product $(0.25 \mathrm{~g}, 60 \%)$, m.p. $>250^{\circ} \mathrm{C}$. ${ }^{1} \mathrm{H}$ NMR (400 MHz, DMSO): $\delta \mathrm{ppm} 2.49\left(\mathrm{~s}, \mathrm{CH}_{3}\right), 6.30$ $(\mathrm{t}, J=6.8 \mathrm{~Hz}, 1 \mathrm{H}), 6.47(\mathrm{~d}, J=9.2 \mathrm{~Hz}, 1 \mathrm{H}), 6.93(\mathrm{~d}, J=3.2 \mathrm{~Hz}$, $1 \mathrm{H}), 7.27(\mathrm{t}, J=6.8 \mathrm{~Hz}, 1 \mathrm{H}), 7.39(\mathrm{~d}, J=8.8 \mathrm{~Hz}, 2 \mathrm{H})$, $7.47-7.64(\mathrm{~m}, 4 \mathrm{H}), 7.82(\mathrm{~d}, J=8.8 \mathrm{~Hz}, 2 \mathrm{H}), 7.93$ (d, $J=8.0 \mathrm{~Hz}, 1 \mathrm{H}), 8.36(\mathrm{~d}, J=8.0 \mathrm{~Hz}, 1 \mathrm{H}), 10.67$ $(\mathrm{s}, \mathrm{NH}), 11.53(\mathrm{~s}, \mathrm{NH})$. HRMS (ESI) calcd. for $\mathrm{C}_{24} \mathrm{H}_{19} \mathrm{~N}_{3} \mathrm{O}_{3} \mathrm{~S}:[\mathrm{M}+\mathrm{Na}]^{+} \mathrm{m} / \mathrm{z}$ : 452.1045, found: 452.1032 . 
5-chloro-N-(2-((4-(2-oxopyridin-1(2H)-yl)phenyl)carbamoyl)phenyl)furan-2-carboxamide (6c)

White solid product $(0.24 \mathrm{~g}, 59 \%)$, m.p. $>250^{\circ} \mathrm{C} .{ }^{1} \mathrm{H}$ NMR (400 MHz, DMSO): $\delta$ ppm $6.31(\mathrm{t}, J=6.4 \mathrm{~Hz}, 1 \mathrm{H})$, $6.47(\mathrm{~d}, J=9.2 \mathrm{~Hz}, 1 \mathrm{H}), 6.76(\mathrm{~d}, J=8.4 \mathrm{~Hz}, 1 \mathrm{H}), 7.33-$ $7.48(\mathrm{~m}, 4 \mathrm{H}), 7.50(\mathrm{t}, J=7.2 \mathrm{~Hz}, 1 \mathrm{H}), 7.62-7.67(\mathrm{~m}, 2 \mathrm{H})$, 7.83 (d, $J=7.6 \mathrm{~Hz}, 2 \mathrm{H}), 7.92$ (d, $J=7.6 \mathrm{~Hz}, 1 \mathrm{H}), 8.34$ $(\mathrm{d}, J=8.0 \mathrm{~Hz}, 1 \mathrm{H}), 10.71$ (s, NH), 11.47 (s, NH). HRMS (ESI) calcd. for $\mathrm{C}_{23} \mathrm{H}_{16} \mathrm{ClN}_{3} \mathrm{O}_{4}:[\mathrm{M}+\mathrm{Na}]^{+} \mathrm{m} / \mathrm{z}: 456.0727$, found: 456.0728 .

5-bromo-N-(2-((4-(2-oxopyridin-1(2H)-yl)phenyl)carbamoyl)phenyl)furan-2-carboxamide (6d)

White solid product $(0.30 \mathrm{~g}, 65 \%)$, m.p. $>250^{\circ} \mathrm{C} .{ }^{1} \mathrm{H}$ NMR (400 MHz, DMSO): $\delta$ ppm $6.31(\mathrm{t}, J=6.4 \mathrm{~Hz}, 1 \mathrm{H})$, $6.47(\mathrm{~d}, J=9.6 \mathrm{~Hz}, 1 \mathrm{H}), 6.85(\mathrm{~d}, J=3.2 \mathrm{~Hz}, 1 \mathrm{H}), 7.29$ $7.33(\mathrm{~m}, 2 \mathrm{H}), 7.41(\mathrm{~d}, J=8.4 \mathrm{~Hz}, 2 \mathrm{H}), 7.48-7.53(\mathrm{~m}, 1 \mathrm{H})$, $7.61(\mathrm{~d}, J=7.6 \mathrm{~Hz}, 1 \mathrm{H}), 7.65(\mathrm{~d}, J=6.4 \mathrm{~Hz}, 1 \mathrm{H}), 7.83$ $(\mathrm{d}, J=8.4 \mathrm{~Hz}, 2 \mathrm{H}), 7.92(\mathrm{~d}, J=7.6 \mathrm{~Hz}, 1 \mathrm{H}), 8.34$ (d, $J=8.0 \mathrm{~Hz}, 1 \mathrm{H}), 10.71(\mathrm{~s}, \mathrm{NH}), 11.46$ (s, NH). HRMS (ESI) calcd. for $\mathrm{C}_{23} \mathrm{H}_{16} \mathrm{BrN}_{3} \mathrm{O}_{4}:[\mathrm{M}+\mathrm{Na}]^{+} \mathrm{m} / \mathrm{z}: 500.0222$, found: 500.0231 .

\section{2-(4-nitrobenzamido)-N-(4-(2-oxopyridin-1}

(2H)-yl)ph-enyl)benzamide (6e)

Yellow solid product $(0.30 \mathrm{~g}, 70 \%)$, m.p. $>250^{\circ} \mathrm{C} .{ }^{1} \mathrm{H}$ NMR (400 MHz, DMSO): $\delta$ ppm 6.30 (t, $J=6.4 \mathrm{~Hz}, 1 \mathrm{H})$, $6.46(\mathrm{~d}, J=9.2 \mathrm{~Hz}, 1 \mathrm{H}), 7.37$ (d, $J=8.8 \mathrm{~Hz}, 3 \mathrm{H}), 7.49$ $(\mathrm{t}, J=8.8 \mathrm{~Hz}, 1 \mathrm{H}), 7.60-7.75(\mathrm{~m}, 2 \mathrm{H}), 7.80(\mathrm{~d}, J=8.8 \mathrm{~Hz}$, 2H), 7.92 (d, $J=7.6 \mathrm{~Hz}, 1 \mathrm{H}), 8.14$ (d, $J=8.4 \mathrm{~Hz}, 2 \mathrm{H})$, 8.29 (d, $J=8.4 \mathrm{~Hz}, 1 \mathrm{H}), 8.39$ (d, $J=8.8 \mathrm{~Hz}, 2 \mathrm{H}), 10.67$ (s, NH), 11.62 (s, NH). HRMS (ESI) calcd. for $\mathrm{C}_{26} \mathrm{H}_{18} \mathrm{~N}_{4} \mathrm{O}_{3}:[\mathrm{M}+\mathrm{Na}]^{+} \mathrm{m} / \mathrm{z}: 477.1175$, found: 477.1165 .

2-(4-cyanobenzamido)-N-(4-(2-oxopyridin-1(2H)-yl) phenyl)benzamide (6f)

Black solid product $(0.24 \mathrm{~g}, 57 \%)$, m.p. $>250^{\circ} \mathrm{C} .{ }^{1} \mathrm{H}$ NMR (400 MHz, DMSO): $\delta$ ppm $6.30(\mathrm{t}, J=6.4 \mathrm{~Hz}, 1 \mathrm{H})$, $6.47(\mathrm{~d}, J=9.2 \mathrm{~Hz}, 1 \mathrm{H}), 7.39-7.32(\mathrm{~m}, 3 \mathrm{H}), 7.49(\mathrm{t}, J=8.8 \mathrm{~Hz}$, $1 \mathrm{H}), 7.62(\mathrm{~d}, J=7.2 \mathrm{~Hz}, 2 \mathrm{H}), 7.80(\mathrm{~d}, J=8.8 \mathrm{~Hz}, 2 \mathrm{H})$, $7.92(\mathrm{~d}, J=8.0 \mathrm{~Hz}, 1 \mathrm{H}), 8.06(\mathrm{~d}, J=8.4 \mathrm{~Hz}, 4 \mathrm{H}), 8.32$ $(\mathrm{d}, J=8.0 \mathrm{~Hz}, 1 \mathrm{H}), 10.69$ (s, NH), 11.60 (s, NH). HRMS (ESI) calcd. for $\mathrm{C}_{26} \mathrm{H}_{18} \mathrm{~N}_{4} \mathrm{O}_{3}:[\mathrm{M}+\mathrm{Na}]^{+} \mathrm{m} / \mathrm{z}: 457.1277$, found: 457.1282 .

4-methoxy-3-nitro-N-(2-((4-(2-oxopyridin-1(2H)-yl)phenyl)carbamoyl)phenyl)benzamide (6g)

White solid product $(0.30 \mathrm{~g}, 65 \%)$, m.p. $>250^{\circ} \mathrm{C}$. ${ }^{1} \mathrm{H}$ NMR (400 MHz, DMSO): $\delta$ ppm $4.0\left(\mathrm{~s}, \mathrm{CH}_{3}\right), 6.30$ $(\mathrm{t}, J=6.8 \mathrm{~Hz}, 1 \mathrm{H}), 6.47(\mathrm{~d}, J=8.8 \mathrm{~Hz}, 1 \mathrm{H}), 7.31-7.39$ (m, 3H), 7.49 (t, $J=8.8 \mathrm{~Hz}, 1 \mathrm{H}), 7.57(\mathrm{~d}, J=9.2 \mathrm{~Hz}, 1 \mathrm{H})$, $7.60-7.64(\mathrm{~m}, 2 \mathrm{H}), 7.81$ (d, $J=8.8 \mathrm{~Hz}, 2 \mathrm{H}), 7.90$ $(\mathrm{d}, J=8.8 \mathrm{~Hz}, 1 \mathrm{H}), 8.17(\mathrm{~d}, J=8.8 \mathrm{~Hz}, 1 \mathrm{H}), 8.24$ (d, $J=8.24 \mathrm{~Hz}, 1 \mathrm{H}), 8.42(\mathrm{~s}, 1 \mathrm{H}), 10.66(\mathrm{~s}, \mathrm{NH}), 11.46$ (s, NH). HRMS (ESI) calcd. for $\mathrm{C}_{26} \mathrm{H}_{20} \mathrm{~N}_{4} \mathrm{O}_{6}:[\mathrm{M}+\mathrm{Na}]^{+}$ $\mathrm{m} / \mathrm{z}$ : 507.1281, found: 507.1281.
6-chloro-N-(2-((4-(2-oxopyridin-1(2H)-yl)phenyl)carbamoyl)phenyl)nicotinamide (6h)

Yellow solid product $(0.26 \mathrm{~g}, 62 \%)$, m.p. $>250^{\circ} \mathrm{C} .{ }^{1} \mathrm{H}$ NMR (400 MHz, DMSO): $\delta$ ppm 6.30 (t, $J=6.4 \mathrm{~Hz}, 1 \mathrm{H})$, $6.46(\mathrm{~d}, J=9.2 \mathrm{~Hz}, 1 \mathrm{H}), 7.33-7.38(\mathrm{~m}, 3 \mathrm{H}), 7.49$ $(\mathrm{t}, J=8.8 \mathrm{~Hz}, 1 \mathrm{H}), 7.60-7.64(\mathrm{~m}, 2 \mathrm{H}), 7.73(\mathrm{~d}, J=8.4 \mathrm{~Hz}, 1 \mathrm{H})$, $7.81(\mathrm{~d}, J=8.4 \mathrm{~Hz}, 2 \mathrm{H}), 7.90(\mathrm{~d}, J=7.6 \mathrm{~Hz}, 1 \mathrm{H})$, $8.22(\mathrm{~d}, J=8.0 \mathrm{~Hz}, 1 \mathrm{H}), 8.28$ (d, $J=8.4 \mathrm{~Hz}, 1 \mathrm{H}), 8.90$ (s, 1H), 10.67 (s, NH), 11.47 (s, NH). HRMS (ESI) calcd. for $\mathrm{C}_{24} \mathrm{H}_{17} \mathrm{ClN}_{4} \mathrm{O}_{3}:[\mathrm{M}+\mathrm{Na}]^{+} \mathrm{m} / \mathrm{z}: 467.0887$, found: 467.0882 .

3,4-difluoro-N-(2-((4-(2-oxopyridin-1(2H)-yl)phenyl) carbamoyl)phenyl)benzamide (6i)

White solid product $(0.24 \mathrm{~g}, 60 \%)$, m.p. $>250^{\circ} \mathrm{C} .{ }^{1} \mathrm{H}$ NMR (400 MHz, DMSO): $\delta$ ppm 6.30 (t, $J=6.4 \mathrm{~Hz}, 1 \mathrm{H})$, $6.46(\mathrm{~d}, J=9.2 \mathrm{~Hz}, 1 \mathrm{H}), 7.33(\mathrm{~d}, J=7.6 \mathrm{~Hz}, 1 \mathrm{H}), 7.38$ $(\mathrm{d}, J=8.4 \mathrm{~Hz}, 2 \mathrm{H}), 7.49(\mathrm{t}, J=8.8 \mathrm{~Hz}, 1 \mathrm{H}), 7.60-7.68(\mathrm{~m}, 3 \mathrm{H})$, $7.80(\mathrm{~d}, J=8.4 \mathrm{~Hz}, 3 \mathrm{H}), 7.90-7.94(\mathrm{~m}, 2 \mathrm{H}), 8.27$ (d, $J=8.4 \mathrm{~Hz}, 1 \mathrm{H}), 10.67$ (s, NH), 11.47 (s, NH). HRMS (ESI) calcd. for $\mathrm{C}_{25} \mathrm{H}_{17} \mathrm{~F}_{2} \mathrm{~N}_{3} \mathrm{O}_{3}:[\mathrm{M}+\mathrm{Na}]^{+} \mathrm{m} / \mathrm{z}: 468.1136$, found: 468.1133 .

3,4,5-trifluoro-N-(2-((4-(2-oxopyridin-1(2H)-yl)phenyl) arbamoyl)phenyl)benzamide (6j)

White solid product $(0.25 \mathrm{~g}, 59 \%)$, m.p. $>250{ }^{\circ} \mathrm{C} .{ }^{1} \mathrm{H}$ NMR (400 MHz, DMSO): $\delta$ ppm 6.38 (t, $J=6.4 \mathrm{~Hz}, 1 \mathrm{H})$, $6.46(\mathrm{~d}, J=8.8 \mathrm{~Hz}, 1 \mathrm{H}), 7.37$ (d, $J=8.8 \mathrm{~Hz}, 3 \mathrm{H}), 7.49$ (t, $J=8.8 \mathrm{~Hz}, 1 \mathrm{H}), 7.62(\mathrm{~d}, J=8.4 \mathrm{~Hz}, 2 \mathrm{H}), 7.79-7.90$ (m, 4H), 7.89 (d, $J=8.0 \mathrm{~Hz}, 1 \mathrm{H}), 8.13$ (d, $J=8.0 \mathrm{~Hz} 1 \mathrm{H})$, 10.65 (s, NH), 11.31 (s, NH). HRMS (ESI) calcd. for $\mathrm{C}_{25} \mathrm{H}_{16} \mathrm{~F}_{3} \mathrm{~N}_{3} \mathrm{O}_{3}:[\mathrm{M}+\mathrm{Na}]^{+} \mathrm{m} / \mathrm{z}: 486.1041$, found: 486.1031 .

2,4-dichloro-N-(2-((4-(2-oxopyridin-1(2H)-yl)phenyl) arbamoyl)phenyl)benzamide (6k)

White solid product $(0.26 \mathrm{~g}, 61 \%)$, m.p. $>250^{\circ} \mathrm{C} .{ }^{1} \mathrm{H}$ NMR (400 MHz, DMSO): $\delta$ ppm 6.30 (t, $J=6.4 \mathrm{~Hz}, 1 \mathrm{H})$, $6.46(\mathrm{~d}, J=9.2 \mathrm{~Hz}, 1 \mathrm{H}), 7.35$ (d, $J=7.6 \mathrm{~Hz}, 3 \mathrm{H}), 8.49$ (t, $J=8.0 \mathrm{~Hz}, 1 \mathrm{H}), 7.56-7.67(\mathrm{~m}, 4 \mathrm{H}), 7.75-7.84(\mathrm{~m}, 4 \mathrm{H})$, $8.14(\mathrm{~d}, J=6.8 \mathrm{~Hz}, 1 \mathrm{H}), 10.64$ (s, NH), 10.99 (s, NH). HRMS (ESI) calcd. for $\mathrm{C}_{25} \mathrm{H}_{17} \mathrm{Cl}_{2} \mathrm{~N}_{3} \mathrm{O}_{3}:[\mathrm{M}+\mathrm{Na}]^{+} \mathrm{m} / \mathrm{z}$ : 500.0545, found: 500.0536 .

2-(4-methoxybenzamido)-N-(4-(2-oxopyridin-1(2H)-yl) phenyl)benzamide (6l)

White solid product $(0.22 \mathrm{~g}, 56 \%)$, m.p. $>250^{\circ} \mathrm{C}$. ${ }^{1} \mathrm{H}$ NMR (400 MHz, DMSO): $\delta$ ppm $3.82\left(\mathrm{~s}, \mathrm{CH}_{3}\right)$, $6.30(\mathrm{t}, J=6.8 \mathrm{~Hz}, 1 \mathrm{H}), 6.47(\mathrm{~d}, J=9.2 \mathrm{~Hz}, 1 \mathrm{H}), 7.10$ $(\mathrm{d}, J=8.8 \mathrm{~Hz}, 2 \mathrm{H}), 7.28(\mathrm{t}, J=7.2 \mathrm{~Hz}, 1 \mathrm{H}), 7.39$ $(\mathrm{d}, J=8.8 \mathrm{~Hz}, 2 \mathrm{H}), 7.50(\mathrm{t}, J=8.8 \mathrm{~Hz}, 1 \mathrm{H}), 7.59-7.64$ $(\mathrm{m}, 2 \mathrm{H}), 7.82(\mathrm{~d}, J=8.8 \mathrm{~Hz}, 2 \mathrm{H}), 7.89$ (d, $J=8.8 \mathrm{~Hz}, 2 \mathrm{H})$, $7.94(\mathrm{~d}, J=7.6,1 \mathrm{H}), 8.49(\mathrm{~d}, J=8.0 \mathrm{~Hz}, 1 \mathrm{H}), 10.69(\mathrm{~s}, \mathrm{NH})$, $11.60(\mathrm{~s}, \mathrm{NH})$. HRMS (ESI) calcd. for $\mathrm{C}_{26} \mathrm{H}_{21} \mathrm{~N}_{3} \mathrm{O}_{4}:[\mathrm{M}+\mathrm{Na}]^{+}$ m/z: 462.1430, found: 462.1430 
3,4-dimethoxy-N-(2-((4-(2-oxopyridin-1(2H)-yl)phenyl) arbamoyl)phenyl)benzamide (6m)

White solid product $(0.24 \mathrm{~g}, 58 \%)$, m.p. $>250^{\circ} \mathrm{C}$. ${ }^{1} \mathrm{H}$ NMR (400 MHz, DMSO): $\delta \mathrm{ppm} 3.83\left(\mathrm{~s}, \mathrm{CH}_{3}\right)$, $6.30(\mathrm{t}, J=6.4 \mathrm{~Hz}, 1 \mathrm{H}), 6.47(\mathrm{~d}, J=9.2 \mathrm{~Hz}, 1 \mathrm{H}), 7.14$ $(\mathrm{d}, J=8.4 \mathrm{~Hz}, 1 \mathrm{H}), 7.28(\mathrm{t}, J=7.6 \mathrm{~Hz}, 1 \mathrm{H}), 7.39$ (t, $J=8.4 \mathrm{~Hz}, 2 \mathrm{H}), 7.48-7.52(\mathrm{~m}, 3 \mathrm{H}), 7.60-7.63(\mathrm{~m}, 2 \mathrm{H})$, $7.84(\mathrm{~d}, J=8.8 \mathrm{~Hz}, 2 \mathrm{H}), 7.92(\mathrm{~d}, J=7.6 \mathrm{~Hz}, 1 \mathrm{H}), 8.47$ (d, $J=8.4 \mathrm{~Hz}, 1 \mathrm{H}), 10.69$ (s, NH), 11.59 (s, NH). HRMS (ESI) calcd. for $\mathrm{C}_{27} \mathrm{H}_{23} \mathrm{~N}_{3} \mathrm{O}_{5}:[\mathrm{M}+\mathrm{Na}]^{+} \mathrm{m} / \mathrm{z}$ : 492.1535, found: 492.1531 .

3,4,5-trimethoxy-N-(2-((4-(2-oxopyridin-1(2H)-yl) phenyl)carbamoyl)phenyl)benzamide (6n)

White solid product $(0.28 \mathrm{~g}, 63 \%)$, m.p. $>250^{\circ} \mathrm{C}$. ${ }^{1} \mathrm{H}$ NMR (400 MHz, DMSO): $\delta$ ppm $3.84\left(\mathrm{~s}, \mathrm{CH}_{3}\right), 3.72$ $\left(\mathrm{s}, \mathrm{CH}_{3}\right), 6.30$ (t, $\left.J=6.4 \mathrm{~Hz}, 1 \mathrm{H}\right), 6.46(\mathrm{~d}, J=9.2 \mathrm{~Hz}, 1 \mathrm{H})$, $7.23(\mathrm{~s}, 2 \mathrm{H}), 7.31(\mathrm{t}, J=8.0 \mathrm{~Hz}, 1 \mathrm{H}), 7.37(\mathrm{~d}, J=8.8 \mathrm{~Hz}, 2 \mathrm{H})$, 7.49 (t, $J=7.2 \mathrm{~Hz}, 1 \mathrm{H}), 7.60-7.61(\mathrm{~m}, 2 \mathrm{H}), 7.86$ $(\mathrm{d}, J=8.4 \mathrm{~Hz}, 2 \mathrm{H}), 7.91(\mathrm{~d}, J=7.6 \mathrm{~Hz}, 1 \mathrm{H}), 8.31$ (d, $J=8.4 \mathrm{~Hz}, 1 \mathrm{H}), 10.65$ (s, NH), $11.42(\mathrm{~s}, \mathrm{NH})$. HRMS (ESI) calcd. for $\mathrm{C}_{28} \mathrm{H}_{25} \mathrm{~N}_{3} \mathrm{O}_{6}:[\mathrm{M}+\mathrm{Na}]^{+} \mathrm{m} / \mathrm{z}: 522.1641$, found: 522.1633 .

N-(4-(2-oxopyridin-1(2H)-yl)phenyl)-2-(4-(trifluoromethyl)benzamido)benzamide (6o)

White solid product $(0.27 \mathrm{~g}, 62 \%)$, m.p. $>250^{\circ} \mathrm{C} .{ }^{1} \mathrm{H}$ NMR (400 MHz, DMSO): $\delta$ ppm $6.30(\mathrm{t}, J=6.8 \mathrm{~Hz}, 1 \mathrm{H})$, $6.46(\mathrm{~d}, J=9.2 \mathrm{~Hz}, 1 \mathrm{H}), 7.33-7.39(\mathrm{~m}, 3 \mathrm{H}), 7.49$ $(\mathrm{t}, J=8.8 \mathrm{~Hz}, 1 \mathrm{H}), 7.61-7.66(\mathrm{~m}, 2 \mathrm{H}), 7.80(\mathrm{~d}, J=8.8 \mathrm{~Hz}, 2 \mathrm{H})$, $7.92-7.96(\mathrm{~m}, 3 \mathrm{H}), 8.10(\mathrm{~d}, J=8.4 \mathrm{~Hz}, 2 \mathrm{H}), 8.34$ $(\mathrm{d}, J=8.4 \mathrm{~Hz}, 1 \mathrm{H}), 10.69(\mathrm{~s}, \mathrm{NH}), 11.62(\mathrm{~s}, \mathrm{NH})$. HRMS (ESI) calcd. for $\mathrm{C}_{26} \mathrm{H}_{18} \mathrm{~F}_{3} \mathrm{~N}_{3} \mathrm{O}_{3}:[\mathrm{M}+\mathrm{Na}]^{+} \mathrm{m} / \mathrm{z}$ : 500.1198 , found: 500.1191

\section{5-bromo-N-(4-chloro-2-((4-(2-oxopyridin-1(2H)-yl)} ph-enyl)carbamoyl)phenyl)thiophene-2-carboxamide (6a-a)

White solid product $(0.30 \mathrm{~g}, 59 \%)$, m.p. $>250^{\circ} \mathrm{C} .{ }^{1} \mathrm{H}$ NMR (400 MHz, DMSO): $\delta$ ppm $6.31(\mathrm{t}, J=6.4 \mathrm{~Hz}, 1 \mathrm{H})$, $6.47(\mathrm{~d}, J=9.2 \mathrm{~Hz}, 1 \mathrm{H}), 7.39(\mathrm{~d}, J=8.8 \mathrm{~Hz}, 3 \mathrm{H}), 7.50$ $(\mathrm{t}, J=8.8 \mathrm{~Hz}, 1 \mathrm{H}), 7.59-7.68(\mathrm{~m}, 2 \mathrm{H}), 7.80(\mathrm{~d}, J=8.4 \mathrm{~Hz}, 2 \mathrm{H})$, $7.96(\mathrm{~s}, 1 \mathrm{H}), 8.20(\mathrm{~d}, J=8.8 \mathrm{~Hz}, 1 \mathrm{H}), 10.73(\mathrm{~s}, \mathrm{NH})$, 11.40 (s, NH). HRMS (ESI) calcd. for $\mathrm{C}_{23} \mathrm{H}_{15} \mathrm{BrClN}_{3} \mathrm{O}_{3} \mathrm{~S}$ : $[\mathrm{M}+\mathrm{Na}]^{+} \mathrm{m} / \mathrm{z}: 549.9604$, found: 549.9588 .

5-bromo-N-(4-methyl-2-((4-(2-oxopyridin-1(2H)-yl)phenyl)carbamoyl)phenyl)thiophene-2-carboxamide (6a-b)

White solid product $(0.26$ g, $54 \%)$, m.p. $>250^{\circ} \mathrm{C}$. ${ }^{1} \mathrm{H}$ NMR (400 MHz, DMSO): $\delta$ ppm $2.39\left(\mathrm{~s}, \mathrm{CH}_{3}\right), 6.31$ $(\mathrm{t}, J=6.4 \mathrm{~Hz}, 1 \mathrm{H}), 6.47(\mathrm{~d}, J=9.2 \mathrm{~Hz}, 1 \mathrm{H}), 7.36-7.43(\mathrm{~m}, 4 \mathrm{H})$, $7.50(\mathrm{t}, J=8.8 \mathrm{~Hz}, 1 \mathrm{H}), 7.57(\mathrm{~d}, J=4.0 \mathrm{~Hz}, 1 \mathrm{H}), 7.63$ $(\mathrm{d}, J=6.8 \mathrm{~Hz}, 1 \mathrm{H}), 7.72(\mathrm{~s}, 1 \mathrm{H}), 7.81(\mathrm{~d}, J=8.8 \mathrm{~Hz}, 2 \mathrm{H})$, 8.09 (d, $J=8.4 \mathrm{~Hz}, 1 \mathrm{H}), 10.62$ (s, NH), 11.37 (s, NH). HRMS (ESI) calcd. for $\mathrm{C}_{24} \mathrm{H}_{18} \mathrm{BrN}_{3} \mathrm{O}_{3} \mathrm{~S}$ : $[\mathrm{M}+\mathrm{Na}]^{+} \mathrm{m} / \mathrm{z}$ : 530.0150, found: 530.0138 .
5-bromo-N-(2-methyl-6-((4-(2-oxopyridin-1(2H)-yl) ph-enyl)carbamoyl)phenyl)thiophene-2-carboxamide (6a-c)

White solid product $(0.25 \mathrm{~g}, 52 \%)$, m.p. $>250^{\circ} \mathrm{C}$. ${ }^{1} \mathrm{H}$ NMR (400 MHz, DMSO): $\delta$ ppm $2.26\left(\mathrm{~s}, \mathrm{CH}_{3}\right), 6.28$ (t, $J=6.4 \mathrm{~Hz}, 1 \mathrm{H}), 6.45$ (d, $J=9.2 \mathrm{~Hz}, 1 \mathrm{H}), 7.30-7.38$ (m, 4H), 7.46-7.75 (m, 3H), 7.59 (d, $J=6.8 \mathrm{~Hz}, 1 \mathrm{H})$, 7.75-7.83 (m, 3H), 10.08 (s, NH), 10.41 (s, NH). HRMS (ESI) calcd. for $\mathrm{C}_{24} \mathrm{H}_{18} \mathrm{BrN}_{3} \mathrm{O}_{3} \mathrm{~S}:[\mathrm{M}+\mathrm{Na}]^{+} \mathrm{m} / \mathrm{z}: 530.0150$, found: 530.0138

5-bromo-N-(2-((4-(3-oxomorpholino)phenyl)carbamoyl)phenyl)thiophene-2-carboxamide (6a-d)

White solid product $(0.30 \mathrm{~g}, 63 \%)$, m.p. $>250^{\circ} \mathrm{C} .{ }^{1} \mathrm{H}$ NMR (400 MHz, DMSO): $\delta$ ppm $3.72\left(\mathrm{t}, J=4.8 \mathrm{~Hz}, \mathrm{CH}_{2}\right.$ ), $3.97\left(\mathrm{t}, J=4.8 \mathrm{~Hz}, \mathrm{CH}_{2}\right), 4.19\left(\mathrm{~s}, \mathrm{CH}_{2}\right), 7.30(\mathrm{t}, J=7.6$ $\mathrm{Hz}, 1 \mathrm{H}), 7.38(\mathrm{~d}, J=8.0 \mathrm{~Hz}, 3 \mathrm{H}), 7.56-7.62(\mathrm{~m}, 2 \mathrm{H})$, $7.72(\mathrm{~d}, J=8.8 \mathrm{~Hz}, 2 \mathrm{H}), 7.91(\mathrm{~d}, J=7.6 \mathrm{~Hz}, 1 \mathrm{H}), 8.25$ (d, $J=8.4 \mathrm{~Hz}, 1 \mathrm{H}), 10.57$ (s, NH), 11.60 (s, NH). HRMS (ESI) calcd. for $\mathrm{C}_{22} \mathrm{H}_{18} \mathrm{BrN}_{3} \mathrm{O}_{4} \mathrm{~S}$ : $[\mathrm{M}+\mathrm{Na}]^{+} \mathrm{m} / \mathrm{z}$ : 522.0099, found: 522.0098 .

5-bromo-N-(4-chloro-2-((4-(3-oxomorpholino)phenyl) carbamoyl)phenyl)thiophene-2-carboxamide (6a-e)

Yellow solid product $(0.29$ g, $57 \%)$, m.p. $>250^{\circ} \mathrm{C}$. ${ }^{1} \mathrm{H}$ NMR (400 MHz, DMSO): $\delta$ ppm $3.72(\mathrm{t}, J=4.8 \mathrm{~Hz}$, $\left.\mathrm{CH}_{2}\right), 3.97$ (t, $\left.J=4.8 \mathrm{~Hz}, \mathrm{CH}_{2}\right), 4.19\left(\mathrm{~s}, \mathrm{CH}_{2}\right), 7.37-7.40$ $(\mathrm{m}, 3 \mathrm{H}), 7.58(\mathrm{~d}, J=4.0 \mathrm{~Hz}, 1 \mathrm{H}), 7.65-7.71(\mathrm{~m}, 3 \mathrm{H}), 7.95$ (s, 1H), 8.22 (d, $J=9.2 \mathrm{~Hz}, 1 \mathrm{H}), 10.63(\mathrm{~s}, \mathrm{NH}), 11.48$ (s, NH). HRMS (ESI) calcd. for $\mathrm{C}_{22} \mathrm{H}_{17} \mathrm{BrClN}_{3} \mathrm{O}_{4} \mathrm{~S}:[\mathrm{M}+$ $\mathrm{Na}]^{+}$m/z: 555.9709, found: 555.9707.

5-bromo-N-(4-methyl-2-((4-(3-oxomorpholino)phenyl) carbamoyl)phenyl)thiophene-2-carboxamide (6a-f)

White solid product $(0.24 \mathrm{~g}, 49 \%)$, m.p. $>250^{\circ} \mathrm{C}$. ${ }^{1} \mathrm{H}$ NMR (400 MHz, DMSO): $\delta$ ppm $2.60\left(\mathrm{~s}, \mathrm{CH}_{3}\right), 3.72$ (t, $\left.J=4.8 \mathrm{~Hz}, \mathrm{CH}_{2}\right), 3.97\left(\mathrm{t}, J=4.8 \mathrm{~Hz}, \mathrm{CH}_{2}\right), 4.19(\mathrm{~s}$, $\left.\mathrm{CH}_{2}\right), 7.47-7.53(\mathrm{~m}, 4 \mathrm{H}), 7.67(\mathrm{~d}, J=4.0 \mathrm{~Hz}, 1 \mathrm{H}), 7.82$ $(\mathrm{d}, J=8.4 \mathrm{~Hz}, 3 \mathrm{H}), 8.23(\mathrm{~d}, J=8.4 \mathrm{~Hz}, 1 \mathrm{H}), 10.64(\mathrm{~s}, \mathrm{NH})$, $11.56(\mathrm{~s}, \mathrm{NH})$. HRMS (ESI) calcd. for $\mathrm{C}_{23} \mathrm{H}_{20} \mathrm{BrN}_{3} \mathrm{O}_{4} \mathrm{~S}$ : $[\mathrm{M}+\mathrm{Na}]^{+} \mathrm{m} / \mathrm{z}: 536.0256$, found: 536.0256 .

5-bromo-N-(2-methyl-6-((4-(3-oxomorpholino)phenyl) carbamoyl)phenyl)thiophene-2-carboxamide (6a-g)

White solid product $(0.27 \mathrm{~g}, 56 \%)$, m.p. $>250^{\circ} \mathrm{C}$. ${ }^{1} \mathrm{H}$ NMR (400 MHz, DMSO): $\delta$ ppm $2.25\left(\mathrm{~s}, \mathrm{CH}_{3}\right), 3.68(\mathrm{t}$, $\left.J=4.8 \mathrm{~Hz}, \mathrm{CH}_{2}\right), 3.95$ (t, $\left.J=4.8 \mathrm{~Hz}, \mathrm{CH}_{2}\right), 4.17\left(\mathrm{~s}, \mathrm{CH}_{2}\right)$, 7.28-7.34 (m, 4H), 7.44-7.48 (m, 2H), $7.66(\mathrm{~d}, J=8.4$ $\mathrm{Hz}, 2 \mathrm{H}), 7.70$ (d, $J=4.0 \mathrm{~Hz}, 1 \mathrm{H}), 10.06$ (s, NH), 10.28 (s, NH). HRMS (ESI) calcd. for $\mathrm{C}_{23} \mathrm{H}_{20} \mathrm{BrN}_{3} \mathrm{O}_{4} \mathrm{~S}$ : $[\mathrm{M}+\mathrm{Na}]^{+} \mathrm{m} / \mathrm{z}: 536.0256$, found: 536.0242 .

6-chloro-N-(4-chloro-2-((4-(2-oxopyridin-1(2H)-yl)phenyl)carbamoyl)phenyl)nicotinamide (6h-a)

White solid product $(0.33$ g, $73 \%)$, m.p. $>250^{\circ} \mathrm{C} .{ }^{1} \mathrm{H}$ NMR (400 MHz, DMSO): $\delta$ ppm $6.30(\mathrm{t}, J=6.8 \mathrm{~Hz}, 1 \mathrm{H})$, $6.46(\mathrm{~d}, J=9.2 \mathrm{~Hz}, 1 \mathrm{H}), 7.37$ (d, $J=8.8 \mathrm{~Hz}, 2 \mathrm{H})$, 
$7.49(\mathrm{t}, J=8.8 \mathrm{~Hz}, 1 \mathrm{H}), 7.62$ (d, $J=8.4 \mathrm{~Hz}, 2 \mathrm{H}), 7.72$ $(\mathrm{d}, J=8.4 \mathrm{~Hz}, 2 \mathrm{H}), 7.81(\mathrm{~d}, J=6.8 \mathrm{~Hz}, 2 \mathrm{H}), 7.94(\mathrm{~s}, 1 \mathrm{H})$, $8.20(\mathrm{~d}, J=8.4 \mathrm{~Hz}, 1 \mathrm{H}), 8.27$ (d, $J=8.0 \mathrm{~Hz}, 1 \mathrm{H}), 8.89$ (s, 1H), 10.73 (s, NH), 11.37 (s, NH). HRMS (ESI) calcd. for $\mathrm{C}_{24} \mathrm{H}_{16} \mathrm{Cl}_{2} \mathrm{~N}_{4} \mathrm{O}_{3}:[\mathrm{M}+\mathrm{Na}]^{+} \mathrm{m} / \mathrm{z}: 501.0497$, found: 501.0494 .

6-chloro-N-(4-methyl-2-((4-(2-oxopyridin-1(2H)-yl)phenyl)carbamoyl)phenyl)nicotinamide (6h-b)

White solid product $(0.29 \mathrm{~g}, 67 \%)$, m.p. $>250^{\circ} \mathrm{C}$. ${ }^{1} \mathrm{H}$ NMR (400 MHz, DMSO): $\delta$ ppm $2.40\left(\mathrm{~s}, \mathrm{CH}_{3}\right), 6.30$ $(\mathrm{t}, J=6.8 \mathrm{~Hz}, 1 \mathrm{H}), 6.46(\mathrm{~d}, J=9.2 \mathrm{~Hz}, 1 \mathrm{H}), 7.37(\mathrm{~d}, J=8.8$ $\mathrm{Hz}, 2 \mathrm{H}), 7.43$ (d, $J=8.0 \mathrm{~Hz}, 1 \mathrm{H}), 7.49$ (t, $J=8.8 \mathrm{~Hz}, 1 \mathrm{H})$, $7.62(\mathrm{~d}, J=6.8 \mathrm{~Hz}, 1 \mathrm{H}), 7.72(\mathrm{~d}, J=8.4 \mathrm{~Hz}, 2 \mathrm{H}), 7.80$ $(\mathrm{d}, J=8.8 \mathrm{~Hz}, 2 \mathrm{H}), 8.08(\mathrm{~d}, J=8.4 \mathrm{~Hz}, 1 \mathrm{H}), 8.26$ (d, $J=8.4 \mathrm{~Hz}, 1 \mathrm{H}), 8.88(\mathrm{~s}, 1 \mathrm{H}), 10.62(\mathrm{~s}, \mathrm{NH}), 11.34$ (s, NH). HRMS (ESI) calcd. for $\mathrm{C}_{25} \mathrm{H}_{19} \mathrm{ClN}_{4} \mathrm{O}_{3}:[\mathrm{M}+\mathrm{Na}]^{+}$ m/z: 481.1043, found: 481.1037.

6-chloro-N-(2-methyl-6-((4-(2-oxopyridin-1(2H)-yl)phenyl)carbamoyl)phenyl)nicotinamide (6h-c)

White solid product $(0.27 \mathrm{~g}, 62 \%), \mathrm{m} . \mathrm{p} .>250^{\circ} \mathrm{C}$. ${ }^{1} \mathrm{H}$ NMR (400 MHz, DMSO): $\delta$ ppm $2.28\left(\mathrm{~s}, \mathrm{CH}_{3}\right)$, $6.28(\mathrm{t}, J=6.8 \mathrm{~Hz}, 1 \mathrm{H}), 6.44(\mathrm{~d}, J=8.8 \mathrm{~Hz}, 1 \mathrm{H}), 7.30$ $(\mathrm{d}, J=8.8 \mathrm{~Hz}, 2 \mathrm{H}), 7.34(\mathrm{~d}, J=8.8 \mathrm{~Hz}, 1 \mathrm{H}), 7.45-7.51$ $(\mathrm{m}, 3 \mathrm{H}), 7.58(\mathrm{~d}, J=8.8 \mathrm{~Hz}, 1 \mathrm{H}), 7.67(\mathrm{~d}, J=8.4 \mathrm{~Hz}, 1 \mathrm{H})$, $7.76(\mathrm{~d}, J=8.4 \mathrm{~Hz}, 2 \mathrm{H}), 8.29$ (d, $J=8.0 \mathrm{~Hz}, 1 \mathrm{H}), 8.89$ (s, 1H), 10.23 (s, NH), 10.47 (s, NH). HRMS (ESI) calcd. for $\mathrm{C}_{25} \mathrm{H}_{19} \mathrm{ClN}_{4} \mathrm{O}_{3}:[\mathrm{M}+\mathrm{Na}]^{+} \mathrm{m} / \mathrm{z}: 481.1043$, found: 481.1039 .

6-chloro-N-(2-((4-(3-oxomorpholino)phenyl)carbamoyl)phenyl)nicotinamide (6h-d)

White solid product $(0.30 \mathrm{~g}, 69 \%)$, m.p. $>250^{\circ} \mathrm{C}$. ${ }^{1} \mathrm{H}$ NMR (400 MHz, DMSO): $\delta$ ppm 3.71 (t, $J=4.8$ $\left.\mathrm{Hz}, \mathrm{CH}_{2}\right), 3.97$ (t, $\left.J=4.8 \mathrm{~Hz}, \mathrm{CH}_{2}\right), 4.19\left(\mathrm{~s}, \mathrm{CH}_{2}\right), 7.36$ $(\mathrm{d}, J=8.8 \mathrm{~Hz}, 3 \mathrm{H}), 7.62(\mathrm{t}, J=7.2 \mathrm{~Hz}, 1 \mathrm{H}), 7.70-7.73$ (m, 3H), 7.89 (d, $J=7.6 \mathrm{~Hz}, 1 \mathrm{H}), 8.24-8.29$ (m, 2H), 8.89 (m, 1H), 10.56 (s, NH), 11.54 (s, NH). HRMS (ESI) calcd. for $\mathrm{C}_{23} \mathrm{H}_{19} \mathrm{ClN}_{4} \mathrm{O}_{4}:[\mathrm{M}+\mathrm{Na}]^{+} \mathrm{m} / \mathrm{z}: 473.0993$, found: 473.0987.

6-chloro-N-(4-chloro-2-((4-(3-oxomorpholino)phenyl) carbamoyl)phenyl)nicotinamide (6h-e)

Yellow solid product $(0.28 \mathrm{~g}, 61 \%), \mathrm{m} . \mathrm{p} .>250^{\circ} \mathrm{C}$. ${ }^{1} \mathrm{H}$ NMR (400 MHz, DMSO): $\delta$ ppm 3.71 (t, $J=4.8$ $\left.\mathrm{Hz}, \mathrm{CH}_{2}\right), 3.97$ (t, $\left.J=4.8 \mathrm{~Hz}, \mathrm{CH}_{2}\right), 4.19\left(\mathrm{~s}, \mathrm{CH}_{2}\right), 7.37$ $(\mathrm{d}, J=8.8 \mathrm{~Hz}, 2 \mathrm{H}), 7.67-7.74(\mathrm{~m}, 4 \mathrm{H}), 7.93(\mathrm{~s}, 1 \mathrm{H}), 8.20$ 8.27 (m, 2H), $8.88(\mathrm{~m}, 1 \mathrm{H}), 10.63$ (s, NH), 11.43 (s, NH). HRMS (ESI) calcd. for $\mathrm{C}_{23} \mathrm{H}_{18} \mathrm{Cl}_{2} \mathrm{~N}_{4} \mathrm{O}_{4}:[\mathrm{M}+\mathrm{Na}]^{+} \mathrm{m} / \mathrm{z}$ : 507.0603, found: 507.0594.

6-chloro-N-(4-methyl-2-((4-(3-oxomorpholino)phenyl) carbamoyl)phenyl)nicotinamide (6h-f)

White solid product $(0.28 \mathrm{~g}, 64 \%)$, m.p. $>250^{\circ} \mathrm{C}$. ${ }^{1} \mathrm{H}$ NMR (400 MHz, DMSO): $\delta$ ppm $2.39\left(\mathrm{~s}, \mathrm{CH}_{3}\right)$, 3.71 (t, $\left.J=4.8 \mathrm{~Hz}, \mathrm{CH}_{2}\right), 3.97$ (t, $J=4.8 \mathrm{~Hz}, \mathrm{CH}_{2}$ ), 4.19 (s, $\left.\mathrm{CH}_{2}\right), 7.36(\mathrm{~d}, J=8.4 \mathrm{~Hz}, 2 \mathrm{H}), 7.43(\mathrm{~d}, J=8.8 \mathrm{~Hz}, 1 \mathrm{H})$, $7.71(\mathrm{~d}, J=8.8 \mathrm{~Hz}, 4 \mathrm{H}), 8.11(\mathrm{~d}, J=8.4 \mathrm{~Hz}, 1 \mathrm{H}), 8.25$ $(\mathrm{d}, J=8.0 \mathrm{~Hz}, 1 \mathrm{H}), 8.88(\mathrm{~s}, 1 \mathrm{H}), 10.52(\mathrm{~s}, \mathrm{NH}), 11.41$ (s, NH). HRMS (ESI) calcd. for $\mathrm{C}_{24} \mathrm{H}_{21} \mathrm{ClN}_{4} \mathrm{O}_{4}:[\mathrm{M}+\mathrm{Na}]^{+}$ $\mathrm{m} / \mathrm{z}$ : 487.1149, found: 487.1148 .

6-chloro-N-(2-methyl-6-((4-(3-oxomorpholino)phenyl) carbamoyl)phenyl)nicotinamide (6h-g)

White solid product $(0.27 \mathrm{~g}, 62 \%)$, m.p. $>250^{\circ} \mathrm{C}$. ${ }^{1} \mathrm{H}$ NMR (400 MHz, DMSO): $\delta$ ppm 2.27 (s, $\mathrm{CH}_{3}$ ), 3.71 $\left(\mathrm{t}, J=4.8 \mathrm{~Hz}, \mathrm{CH}_{2}\right), 3.97\left(\mathrm{t}, J=4.8 \mathrm{~Hz}, \mathrm{CH}_{2}\right), 4.17$ $\left(\mathrm{s}, \mathrm{CH}_{2}\right), 7.27-7.34(\mathrm{~m}, 3 \mathrm{H}), 7.45-7.51(\mathrm{~m}, 2 \mathrm{H}), 7.65-7.73$ $(\mathrm{m}, 3 \mathrm{H}), 8.28(\mathrm{~d}, J=8.0 \mathrm{~Hz}, 1 \mathrm{H}), 8.89(\mathrm{~s}, 1 \mathrm{H}), 10.10$ $(\mathrm{s}, \mathrm{NH}), 10.21(\mathrm{~s}, \mathrm{NH})$. HRMS (ESI) calcd. for $\mathrm{C}_{24} \mathrm{H}_{21} \mathrm{ClN}_{4} \mathrm{O}_{4}:[\mathrm{M}+\mathrm{Na}]^{+} \mathrm{m} / \mathrm{z}: 487.1149$, found: 487.1146 .

2,4-dichloro-N-(4-chloro-2-((4-(2-oxopyridin-1(2H)-yl) phenyl)carbamoyl)phenyl)benzamide (6k-a)

White solid product $(0.27 \mathrm{~g}, 59 \%)$, m.p. $>250^{\circ} \mathrm{C} .{ }^{1} \mathrm{H}$ NMR (400 MHz, DMSO): $\delta$ ppm 6.29 (t, $J=6.8 \mathrm{~Hz}, 1 \mathrm{H})$, $6.46(\mathrm{~d}, J=9.2 \mathrm{~Hz}, 1 \mathrm{H}), 7.36(\mathrm{~d}, J=8.0 \mathrm{~Hz}, 2 \mathrm{H})$, 7.49 (t, $J=8.8 \mathrm{~Hz}, 1 \mathrm{H}), 7.56-7.61(\mathrm{~m}, 2 \mathrm{H}), 7.64-7.68$ $(\mathrm{m}, 2 \mathrm{H}), 7.74-7.79(\mathrm{~m}, 2 \mathrm{H}), 7.87(\mathrm{~s}, 1 \mathrm{H}), 8.10(\mathrm{~d}, J=7.6 \mathrm{~Hz}$, 1H), 10.69 (s, NH), 10.94 (s, NH). HRMS (ESI) calcd. for $\mathrm{C}_{25} \mathrm{H}_{16} \mathrm{Cl}_{3} \mathrm{~N}_{3} \mathrm{O}_{3}:[\mathrm{M}+\mathrm{Na}]^{+} \mathrm{m} / \mathrm{z}: 534.0155$, found: 534.0146.

2,4-dichloro-N-(4-methyl-2-((4-(2-oxopyridin-1(2H)-yl) phenyl)carbamoyl)phenyl)benzamide (6k-b)

Yellow solid product $(0.27 \mathrm{~g}, 60 \%)$, m.p. $>250^{\circ} \mathrm{C}$. ${ }^{1} \mathrm{H}$ NMR (400 MHz, DMSO): $\delta$ ppm $2.39\left(\mathrm{~s}, \mathrm{CH}_{3}\right)$, $6.29(\mathrm{t}, J=6.8 \mathrm{~Hz}, 1 \mathrm{H}), 6.46(\mathrm{~d}, J=8.8 \mathrm{~Hz}, 1 \mathrm{H}), 7.35$ (d, $J=8.8 \mathrm{~Hz}, 2 \mathrm{H}), 7.42(\mathrm{~d}, J=8.4 \mathrm{~Hz}, 1 \mathrm{H}), 7.49$ $(\mathrm{t}, J=8.8 \mathrm{~Hz}, 1 \mathrm{H}), 7.55(\mathrm{~d}, J=8.0 \mathrm{~Hz}, 1 \mathrm{H}), 7.59-7.65$ $(\mathrm{m}, 3 \mathrm{H}), 7.73(\mathrm{~s}, 1 \mathrm{H}), 7.79(\mathrm{~d}, J=8.8 \mathrm{~Hz}, 2 \mathrm{H}), 8.00$ (d, $J=8.4 \mathrm{~Hz}, 1 \mathrm{H}), 10.58$ (s, NH), 10.85 (s, NH). HRMS (ESI) calcd. for $\mathrm{C}_{26} \mathrm{H}_{19} \mathrm{Cl}_{2} \mathrm{~N}_{3} \mathrm{O}_{3}:[\mathrm{M}+\mathrm{Na}]^{+} \mathrm{m} / \mathrm{z}: 514.0701$, found: 514.0697 .

2-(2,4-dichlorobenzamido)-3-methyl-N-(4-(2-oxopyridin-1(2H)-yl)phenyl)benzamide (6k-c)

White solid product $(0.26 \mathrm{~g}, 58 \%)$, m.p. $>250^{\circ} \mathrm{C}$. ${ }^{1} \mathrm{H}$ NMR (400 MHz, DMSO): $\delta$ ppm $2.34\left(\mathrm{~s}, \mathrm{CH}_{3}\right), 6.30$ $(\mathrm{t}, J=6.8 \mathrm{~Hz}, 1 \mathrm{H}), 6.46(\mathrm{~d}, J=9.2 \mathrm{~Hz}, 1 \mathrm{H}), 7.34$ $(\mathrm{d}, J=8.8 \mathrm{~Hz}, 3 \mathrm{H}), 7.49-7.57(\mathrm{~m}, 5 \mathrm{H}), 7.61(\mathrm{~d}, J=8.4 \mathrm{~Hz}$, 1H), 7.69 (s, 1H), 7.85 (d, $J=8.4 \mathrm{~Hz}, 2 \mathrm{H}), 10.11$ (s, NH), 10.48 (s, NH). HRMS (ESI) calcd. for $\mathrm{C}_{26} \mathrm{H}_{19} \mathrm{Cl}_{2} \mathrm{~N}_{3} \mathrm{O}_{3}$ : $[\mathrm{M}+\mathrm{Na}]^{+} \mathrm{m} / \mathrm{z}: 514.0701$, found: 514.0699.

\section{2,4-dichloro-N-(2-((4-(3-oxomorpholino)phenyl)carba- moyl)phenyl)benzamide (6k-d)}

White solid product $(0.31 \mathrm{~g}, 71 \%)$, m.p. $>250{ }^{\circ} \mathrm{C} .{ }^{1} \mathrm{H}$ NMR (400 MHz, DMSO): $\delta$ ppm 3.69 (t, $J=4.8 \mathrm{~Hz}, \mathrm{CH}_{2}$ ), $3.96\left(\mathrm{t}, J=4.8 \mathrm{~Hz}, \mathrm{CH}_{2}\right), 4.18\left(\mathrm{~s}, \mathrm{CH}_{2}\right), 7.33(\mathrm{~d}, J=8.4 \mathrm{~Hz}$, $3 \mathrm{H}), 7.55-7.61(\mathrm{~m}, 2 \mathrm{H}), 7.65-7.74(\mathrm{~m}, 4 \mathrm{H}), 7.82$ $(\mathrm{d}, J=8.0 \mathrm{~Hz}, 1 \mathrm{H}), 8.17(\mathrm{~d}, J=7.2 \mathrm{~Hz}, 1 \mathrm{H}), 10.51(\mathrm{~s}, \mathrm{NH})$, $11.02(\mathrm{~s}, \mathrm{NH})$. HRMS (ESI) calcd. for $\mathrm{C}_{24} \mathrm{H}_{19} \mathrm{Cl}_{2} \mathrm{~N}_{3} \mathrm{O}_{4}$ : $[\mathrm{M}+\mathrm{Na}]^{+} \mathrm{m} / \mathrm{z}: 506.0650$, found: 506.0646. 
2,4-dichloro-N-(4-chloro-2-((4-(3-oxomorpholino)phenyl)carbamoyl)phenyl)benzamide (6k-e)

Yellow solid product $(0.29 \mathrm{~g}, 62 \%)$, m.p. $>250^{\circ} \mathrm{C}$. ${ }^{1} \mathrm{H}$ NMR (400 MHz, DMSO): $\delta$ ppm 3.70 (t, $J=4.8 \mathrm{~Hz}$, $\left.\mathrm{CH}_{2}\right), 3.96\left(\mathrm{t}, J=4.8 \mathrm{~Hz}, \mathrm{CH}_{2}\right), 4.18\left(\mathrm{~s}, \mathrm{CH}_{2}\right), 7.35$ $(\mathrm{d}, J=8.8 \mathrm{~Hz}, 2 \mathrm{H}), 7.57(\mathrm{~d}, J=8.4 \mathrm{~Hz}, 1 \mathrm{H}), 7.64-7.70$ $(\mathrm{m}, 4 \mathrm{H}), 7.74(\mathrm{~s}, 1 \mathrm{H}), 7.86(\mathrm{~s}, 1 \mathrm{H}), 8.13(\mathrm{~d}, J=8.8 \mathrm{~Hz}, 1 \mathrm{H})$, $10.59(\mathrm{~s}, \mathrm{NH}), 10.97$ (s, NH). HRMS (ESI) calcd. for $\mathrm{C}_{24} \mathrm{H}_{18} \mathrm{Cl}_{3} \mathrm{~N}_{3} \mathrm{O}_{4}:[\mathrm{M}+\mathrm{Na}]^{+} \mathrm{m} / \mathrm{z}: 540.0261$, found: 540.0269 .

2,4-dichloro-N-(4-methyl-2-((4-(3-oxomorpholino)phenyl)carbamoyl)phenyl)benzamide (6k-f)

Yellow solid product $(0.31 \mathrm{~g}, 70 \%)$, m.p. $>250^{\circ} \mathrm{C}$. ${ }^{1} \mathrm{H}$ NMR (400 MHz, DMSO): $\delta$ ppm 3.69 (t, $J=4.8 \mathrm{~Hz}$, $\left.\mathrm{CH}_{2}\right), 3.96\left(\mathrm{t}, J=4.8 \mathrm{~Hz}, \mathrm{CH}_{2}\right), 4.18\left(\mathrm{~s}, \mathrm{CH}_{2}\right), 7.34$ $(\mathrm{d}, J=8.8 \mathrm{~Hz}, 2 \mathrm{H}), 7.41(\mathrm{~d}, J=8.0 \mathrm{~Hz}, 1 \mathrm{H}), 7.55$ (d, J = 8.4 Hz, 1H), 7.63-7.73 (m, 4H), 8.05 (d, J=8.4 Hz, $1 \mathrm{H}), 10.47$ (s, NH), 10.89 (s, NH). HRMS (ESI) calcd. for $\mathrm{C}_{25} \mathrm{H}_{21} \mathrm{Cl}_{2} \mathrm{~N}_{3} \mathrm{O}_{4}:[\mathrm{M}+\mathrm{Na}]^{+} \mathrm{m} / \mathrm{z}: 520.0807$, found: 520.0802 .

2-(2,4-dichlorobenzamido)-3-methyl-N-(4-(3-oxomorpholino)phenyl)benzamide (6k-g)

White solid product $(0.31 \mathrm{~g}, 69 \%)$, m.p. $>250^{\circ} \mathrm{C} .{ }^{1} \mathrm{H}$ NMR (400 MHz, DMSO): $\delta$ ppm 3.70 (t, $J=4.8 \mathrm{~Hz}, \mathrm{CH}_{2}$ ), $3.96\left(\mathrm{t}, J=4.8 \mathrm{~Hz}, \mathrm{CH}_{2}\right), 4.18\left(\mathrm{~s}, \mathrm{CH}_{2}\right), 7.31-7.35$ (m, 3H), 7.44 (d, $J=7.6 \mathrm{~Hz}, 2 \mathrm{H}), 7.50-7.56(\mathrm{~m}, 2 \mathrm{H}), 7.68$ (s, 1H), 7.75 (d, $J=8.8 \mathrm{~Hz}, 2 \mathrm{H}), 10.08(\mathrm{~s}, \mathrm{NH}), 10.35$ (s, NH). HRMS (ESI) calcd. for $\mathrm{C}_{25} \mathrm{H}_{21} \mathrm{Cl}_{2} \mathrm{~N}_{3} \mathrm{O}_{4}:[\mathrm{M}+\mathrm{Na}]^{+}$ $\mathrm{m} / \mathrm{z}$ : 520.0807, found: 520.0800 .

5-chloro-2-(4-methoxybenzamido)-N-(4-(2-oxopyridin1(2H)-yl)phenyl)benzamide (6l-a)

White solid product $(0.24 \mathrm{~g}, 57 \%)$, m.p. $>250^{\circ} \mathrm{C}$. ${ }^{1} \mathrm{H}$ NMR (400 MHz, DMSO): $\delta$ ppm $3.82\left(\mathrm{~s}, \mathrm{CH}_{3}\right)$, $6.31(\mathrm{t}, J=6.4 \mathrm{~Hz}, 1 \mathrm{H}), 6.47(\mathrm{~d}, J=9.6 \mathrm{~Hz}, 1 \mathrm{H}), 7.10$ $(\mathrm{d}, J=8.8 \mathrm{~Hz}, 2 \mathrm{H}), 7.40(\mathrm{~d}, J=8.8 \mathrm{~Hz}, 2 \mathrm{H}), 7.50$ $(\mathrm{t}, J=8.8 \mathrm{~Hz}, 1 \mathrm{H}), 7.62-7.68(\mathrm{~m}, 2 \mathrm{H}), 7.80(\mathrm{~d}, J=8.4$ $\mathrm{Hz}, 2 \mathrm{H}), 7.88(\mathrm{~d}, J=8.8 \mathrm{~Hz}, 2 \mathrm{H}), 7.99(\mathrm{~s}, 1 \mathrm{H}), 8.46$ (d, $J=9.2 \mathrm{~Hz}, 1 \mathrm{H}), 10.75$ (s, NH), 11.49 (s, NH). HRMS (ESI) calcd. for $\mathrm{C}_{26} \mathrm{H}_{20} \mathrm{ClN}_{3} \mathrm{O}_{4}:[\mathrm{M}+\mathrm{Na}]^{+} \mathrm{m} / \mathrm{z}: 496.1040$, found: 496.1037 .

2-(4-methoxybenzamido)-5-methyl-N-(4-(2-oxopyridin-1(2H)-yl)phenyl)benzamide (61-b)

White solid product $(0.29 \mathrm{~g}, 71 \%)$, m.p. $>250^{\circ} \mathrm{C}$. ${ }^{1} \mathrm{H}$ NMR (400 MHz, DMSO): $\delta$ ppm 2.39 (s, $\mathrm{CH}_{3}$ ), 3.82 $\left(\mathrm{s}, \mathrm{CH}_{3}\right), 6.31(\mathrm{t}, J=6.4 \mathrm{~Hz}, 1 \mathrm{H}), 6.47(\mathrm{~d}, J=9.2 \mathrm{~Hz}, 1 \mathrm{H}), 7.10$ $(\mathrm{d}, J=8.8 \mathrm{~Hz}, 2 \mathrm{H}), 7.38-7.44(\mathrm{~m}, 3 \mathrm{H}), 7.50$ (t, $J=7.2 \mathrm{~Hz}$, $1 \mathrm{H}), 7.63(\mathrm{~d}, J=6.0 \mathrm{~Hz}, 1 \mathrm{H}), 7.75(\mathrm{~s}, 1 \mathrm{H}), 7.81$ (d, $J=8.4 \mathrm{~Hz}, 2 \mathrm{H}), 7.87(\mathrm{~d}, J=8.4 \mathrm{~Hz}, 2 \mathrm{H}), 8.35$ (d, $J=8.4 \mathrm{~Hz}, 2 \mathrm{H}), 10.65$ (s, NH), 11.44 (s, NH). HRMS (ESI) calcd. for $\mathrm{C}_{27} \mathrm{H}_{23} \mathrm{~N}_{3} \mathrm{O}_{4}:[\mathrm{M}+\mathrm{Na}]^{+} \mathrm{m} / \mathrm{z}$ : 476.1586, found: 476.1582 .
2-(4-methoxybenzamido)-3-methyl-N-(4-(2-oxopyridin-1(2H)-yl)phenyl)benzamide (6l-c)

White solid product $(0.30$ g, $73 \%)$, m.p. $>250^{\circ} \mathrm{C}$. ${ }^{1} \mathrm{H}$ NMR (400 MHz, DMSO): $\delta$ ppm $2.26\left(\mathrm{~s}, \mathrm{CH}_{3}\right), 3.81$ $\left(\mathrm{s}, \mathrm{CH}_{3}\right), 6.27(\mathrm{t}, J=6.8 \mathrm{~Hz}, 1 \mathrm{H}), 6.44(\mathrm{~d}, J=9.2 \mathrm{~Hz}, 1 \mathrm{H})$, $7.01(\mathrm{~d}, J=8.8 \mathrm{~Hz}, 2 \mathrm{H}), 7.28-7.36(\mathrm{~m}, 3 \mathrm{H}), 7.45-7.49$ $(\mathrm{m}, 3 \mathrm{H}), 7.57(\mathrm{~d}, J=6.4 \mathrm{~Hz}, 1 \mathrm{H}), 7.76(\mathrm{~d}, J=8.8 \mathrm{~Hz}$, $2 \mathrm{H}), 7.92(\mathrm{~d}, J=8.4 \mathrm{~Hz}, 2 \mathrm{H}), 9.85(\mathrm{~s}, \mathrm{NH}), 10.38$ (s, NH). HRMS (ESI) calcd. for $\mathrm{C}_{27} \mathrm{H}_{23} \mathrm{~N}_{3} \mathrm{O}_{4}:[\mathrm{M}+\mathrm{Na}]^{+}$ $\mathrm{m} / \mathrm{z}$ : 476.1586, found: 476.1585 .

2-(4-methoxybenzamido)-N-(4-(3-oxomorpholino)phenyl)benzamide (6l-d)

White solid product $(0.29 \mathrm{~g}, 72 \%)$, m.p. $>250^{\circ} \mathrm{C}$. ${ }^{1} \mathrm{H}$ NMR (400 MHz, DMSO): $\delta$ ppm $3.72(\mathrm{t}, J=4.8$ $\left.\mathrm{Hz}, \mathrm{CH}_{2}\right), 3.83\left(\mathrm{~s}, \mathrm{CH}_{3}\right), 3.97\left(\mathrm{t}, J=4.8 \mathrm{~Hz}, \mathrm{CH}_{2}\right), 4.19$ $\left(\mathrm{s}, \mathrm{CH}_{2}\right), 7.10(\mathrm{~d}, J=8.4 \mathrm{~Hz}, 2 \mathrm{H}), 7.26(\mathrm{t}, J=7.6 \mathrm{~Hz}, 1 \mathrm{H})$, $7.38(\mathrm{~d}, J=8.8 \mathrm{~Hz}, 2 \mathrm{H}), 7.60(\mathrm{t}, J=8.0 \mathrm{~Hz}, 1 \mathrm{H}), 7.72$ $(\mathrm{d}, J=8.8 \mathrm{~Hz}, 2 \mathrm{H}), 7.87-7.93(\mathrm{~m}, 3 \mathrm{H}), 8.50(\mathrm{~d}, J=8.0 \mathrm{~Hz}, 1 \mathrm{H})$, $10.58(\mathrm{~s}, \mathrm{NH}), 11.64(\mathrm{~s}, \mathrm{NH})$. HRMS (ESI) calcd. for $\mathrm{C}_{25} \mathrm{H}_{23} \mathrm{~N}_{3} \mathrm{O}_{5}:[\mathrm{M}+\mathrm{Na}]^{+} \mathrm{m} / \mathrm{z}: 468.1535$, found: 468.1535 .

5-chloro-2-(4-methoxybenzamido)-N-(4-(3-oxomorpholino)phenyl)benzamide (61-e)

Yellow solid product $(0.23 \mathrm{~g}, 54 \%)$, m.p. $>250^{\circ} \mathrm{C} .{ }^{1} \mathrm{H}$ NMR (400 MHz, DMSO): $\delta$ ppm 3.72 (t, $J=4.8 \mathrm{~Hz}, \mathrm{CH}_{2}$ ), $3.82\left(\mathrm{~s}, \mathrm{CH}_{3}\right), 3.97\left(\mathrm{t}, J=4.8 \mathrm{~Hz}, \mathrm{CH}_{2}\right), 4.19\left(\mathrm{~s}, \mathrm{CH}_{2}\right), 7.10$ (d, $J=8.8 \mathrm{~Hz}, 2 \mathrm{H}), 7.39$ (d, $J=8.8 \mathrm{~Hz}, 2 \mathrm{H}), 7.65-7.62$ (m, 3H), 7.87 (d, $J=8.8 \mathrm{~Hz}, 2 \mathrm{H}), 7.97(\mathrm{~s}, 1 \mathrm{H}), 7.48$ (d, $J=8.8 \mathrm{~Hz}, 1 \mathrm{H}), 10.66$ (s, NH), 11.53 (s, NH). HRMS (ESI) calcd. for $\mathrm{C}_{25} \mathrm{H}_{22} \mathrm{ClN}_{3} \mathrm{O}_{5}:[\mathrm{M}+\mathrm{Na}]^{+} \mathrm{m} / \mathrm{z}$ : 502.1146, found: 502.1144 .

2-(4-methoxybenzamido)-5-methyl-N-(4-(3-oxomorpholino)phenyl)benzamide (6l-f)

White solid product $(0.28 \mathrm{~g}, 68 \%)$, m.p. $>250^{\circ} \mathrm{C}$. ${ }^{1} \mathrm{H}$ NMR (400 MHz, DMSO): $\delta$ ppm 3.72 (t, $J=4.8$ $\left.\mathrm{Hz}, \mathrm{CH}_{2}\right), 3.82\left(\mathrm{~s}, \mathrm{CH}_{3}\right), 3.97$ (t, $\left.J=4.8 \mathrm{~Hz}, \mathrm{CH}_{2}\right), 4.19$ $\left(\mathrm{s}, \mathrm{CH}_{2}\right), 7.09(\mathrm{~d}, J=8.8 \mathrm{~Hz}, 2 \mathrm{H}), 7.37-7.43(\mathrm{~m}, 3 \mathrm{H})$, $7.70-7.73(\mathrm{~m}, 3 \mathrm{H}), 7.86(\mathrm{~d}, J=8.8 \mathrm{~Hz}, 2 \mathrm{H}), 8.37$ (d, $J=8.4 \mathrm{~Hz}, 1 \mathrm{H}), 10.54$ (s, NH), 11.49 (s, NH). HRMS (ESI) calcd. for $\mathrm{C}_{26} \mathrm{H}_{25} \mathrm{~N}_{3} \mathrm{O}_{5}:[\mathrm{M}+\mathrm{Na}]^{+} \mathrm{m} / \mathrm{z}: 482.1692$, found: 482.1686

2-(4-methoxybenzamido)-3-methyl-N-(4-(3-oxomorpholino)phenyl)benzamide (6l-g)

White solid product $(0.29$ g, $70 \%)$, m.p. $>250^{\circ} \mathrm{C}$. ${ }^{1} \mathrm{H}$ NMR (400 MHz, DMSO): $\delta$ ppm $3.66(\mathrm{t}, J=4.8$ $\left.\mathrm{Hz}, \mathrm{CH}_{2}\right), 3.80\left(\mathrm{~s}, \mathrm{CH}_{3}\right), 3.94\left(\mathrm{t}, J=4.8 \mathrm{~Hz}, \mathrm{CH}_{2}\right), 4.16$ $\left(\mathrm{s}, \mathrm{CH}_{2}\right), 7.01(\mathrm{~d}, J=8.8 \mathrm{~Hz}, 2 \mathrm{H}), 7.27-7.32(\mathrm{~m}, 3 \mathrm{H})$, $7.44(\mathrm{~d}, J=8.4 \mathrm{~Hz}, 2 \mathrm{H}), 7.66(\mathrm{~d}, J=8.8 \mathrm{~Hz}, 2 \mathrm{H}), 7.91$ (d, $J=8.4 \mathrm{~Hz}, 2 \mathrm{H}), 9.85(\mathrm{~s}, \mathrm{NH}), 10.26(\mathrm{~s}, \mathrm{NH}) . \mathrm{HRMS}$ (ESI) calcd. for $\mathrm{C}_{26} \mathrm{H}_{25} \mathrm{~N}_{3} \mathrm{O}_{5}:[\mathrm{M}+\mathrm{Na}]^{+} \mathrm{m} / \mathrm{z}$ : 482.1692, found: 482.1683 


\section{Inhibition activity measurement against FXa}

The inhibition of FXa was measured using human FXa (Hyphen BioMed, city, Paris, FRA) and chromogenic substrate CS-11(22) (Hyphen BioMed, Paris, FRA) in 384well microtiter plates at room temperature. The synthesized compounds 6 and rivaroxaban were dissolved in DMSO at a concentration of $10 \mathrm{mM}$ and then serially diluted to spanning a range of $30 \mathrm{nM}$ to $100 \mu \mathrm{M}$, respectively. $2 \mu \mathrm{L}$ of FXa (56.8 nM), $16 \mu \mathrm{L}$ of Tris buffer (adjust to $\mathrm{pH} 7.4$ with $\mathrm{HCl}$ containing $0.3 \mathrm{M} \mathrm{NaCl}$ and $50 \mathrm{mM}$ Tris) and $3 \mu \mathrm{L}$ of test compound were added to the well, respectively. The negative control was composed of the same mixed solutions except replacing test compound with DMSO. The positive control was composed of the same mixed solutions except replacing test compound with rivaroxaban. After incubated at $37^{\circ} \mathrm{C}$ for $5 \mathrm{~min}, 8 \mu \mathrm{L}$ of FXa substrate solution $(3.5 \mathrm{mM})$ was added and then incubated at $37^{\circ} \mathrm{C}$ for $25 \mathrm{~min}$. The FXa activity was measured at $405 \mathrm{~nm}$ using a SpectraMax M5 (Molecular Devices, Sunnyvale, CA, USA). The $\mathrm{IC}_{50}$ was calculated by the software named SPSS (IBM, North Castle, NY, USA) and the Probit function in it.

\section{Thrombin inhibition activity of $6 a, 6 a-b, 6 a-e$, $6 \mathrm{k}, 6 \mathrm{k}-\mathrm{a}$ and $6 \mathrm{k}-\mathrm{b}$}

The inhibition of thrombin was measured using human FIIa (Hyphen BioMed, Paris, FRA) and chromogenic substrate CS-01(38) (Hyphen BioMed, Paris, FRA) in 384-well microtiter plates at room temperature. The compounds 6a, 6a-b, 6a-e, 6k, 6k-a, 6k-b and rivaroxaban were dissolved in DMSO to a concentration of $10 \mathrm{mM}$ and then serially diluted to spanning a range of $10 \mu \mathrm{M}$ to $100 \mu \mathrm{M}$, respectively. $2 \mu \mathrm{L}$ of FIIa $(3 \mathrm{NIH} / \mathrm{mL})$, $20 \mu \mathrm{L}$ of Tris buffer (adjust to $\mathrm{pH} 7.4$ with $\mathrm{HCl}$ ) containing $0.3 \mathrm{M} \mathrm{NaCl}$ and $50 \mathrm{mM}$ Tris and $2 \mu \mathrm{L}$ of test compound were added to the well, respectively. The negative control was composed of the same mixed solutions except replacing test compound with DMSO. The positive control was composed of the same mixed solutions except replacing test compound with rivaroxaban. After incubated at $37^{\circ} \mathrm{C}$ for $5 \mathrm{~min}, 3 \mu \mathrm{L}$ of FIIa substrate solution $(4 \mathrm{mM})$ was added and then incubated at $37^{\circ} \mathrm{C}$ for $25 \mathrm{~min}$. The FIIa activity was measured at $405 \mathrm{~nm}$ using a SpectraMax M5 (Molecular Devices).

\section{Prothrombin time (PT) assay}

A commercially available automatic coagulometer (Steellex Science Instrument Co., Ltd., Beijing, China) was employed to measure PT. The clotting times were also measured using the instrument itself, in accordance with the manufacturer's instructions. Increasing concentrations of inhibitor or solvent were added to human (39 Years old, male, Chinese) plasma and incubated for $3 \mathrm{~min}$ at $37^{\circ} \mathrm{C}$. Prothrombin time (PT) was determined by automatic coagulometer.

\section{Docking simulation}

FXa structure was selected from the protein data bank (PDB code: 2xbv) and prepared using Protein Preparation Wizard in Schrödinger package, including assigning bond orders, adding hydrogen atoms, deleting water molecules, creating disulfide bonds and capping terminals. The original ligand of the protein structure$\mathrm{XBV}$ was used as the docking center to generate the receptor grid parameters. The box size was set as $12 \AA$. Compounds 6a, 6a-b, 6a-e, 6k, 6k-a and 6k-b were prepared using the LigPrep module in Schrödinger. Epik method was used to determine possible ionization state of ligands at $\mathrm{pH} 7.0 \pm 2.0$ and low-energy conformers were produced using OPLS-2005 force field. Molecular docking calculations were performed by using Glide module with default parameters at standard precision in Schrödinger.

\section{CONCLUSIONS}

In conclusion, the synthetic anthranilamide compounds were evaluated as novel inhibitors of FXa. Among 43 compounds, 6a, 6a-b, 6a-e, 6k, 6k-a and 6k-b showed excellent activity and selectivity over thrombin. In further, the compound 6a-e showed best anticoagulant activity $(3.8 \mu \mathrm{M})$ in the six compounds significantly. The computational docking simulation study clarified the interactions mode of compounds. The results in this study indicated that compound 6a-e exhibited remarkable thrombin inhibitory effect in intro and it might be a potent novel anti-coagulator for further in vivo studies.

\section{ACKNOWLEDGMENTS AND FUNDING}

This project was supported by the Scientific and Technological Plan Projects of Tianjin (No. 12ZCZDSY01100).

\section{CONFLICTS OF INTEREST}

The authors confirm that this article content has no conflicts of interest.

\section{REFERENCES}

1. Turpie AG. Advances in oral anticoagulation treatment: the safety and efficacy of rivaroxaban in the prevention and treatment of thromboembolism. Ther Adv Hematol. 2012; 3:309-23.

2. Meierhenrich R, Steinhilber E, Eggermann C, Weiss M, Voglic S, Bögelein D, Gauss A, Georgieff M, Stahl W. Incidence and prognostic impact of new-onset atrial fibrillation in patients with septic shock: a prospective observational study. Crit Care. 2010; 14:R108.

3. Orwat MJ, Qiao JX, He K, Rendina AR, Luettgen JM, Rossi KA, Xin B, Knabb RM, Wexler RR, Lam PY, 
Pinto DJ. Orally bioavailable factor Xa inhibitors containing alpha-substituted gem-dimethyl P4 moieties. Bioorg Med Chem Lett. 2014; 24:3341-45.

4. Fareed J, Thethi I, Hoppensteadt D. Old versus new oral anticoagulants: focus on pharmacology. Annu Rev Pharmacol Toxicol. 2012; 52:79-99.

5. Bassand JP. Review of atrial fibrillation outcome trials of oral anticoagulant and antiplatelet agents. Europace. 2012; $14: 312-24$.

6. Ansell J, Hirsh J, Hylek E, Jacobson A, Crowther M, Palareti G, and American College of Chest Physicians. Pharmacology and management of the vitamin $\mathrm{K}$ antagonists: American College of Chest Physicians Evidence-Based Clinical Practice Guidelines (8th Edition). Chest. 2008; 133:160S-98S.

7. Leadley RJ Jr. Coagulation factor Xa inhibition: biological background and rationale. Curr Top Med Chem. 2001; 1:151-59.

8. Xue T, Ding S, Guo B, Zhou Y, Sun P, Wang H, Chu W, Gong G, Wang Y, Chen X, Yang Y. Design, synthesis, and structure-activity and structure-pharmacokinetic relationship studies of novel [6,6,5] tricyclic fused oxazolidinones leading to the discovery of a potent, selective, and orally bioavailable FXa inhibitor. J Med Chem. 2014; 57:7770-91.

9. Maignan S, Guilloteau JP, Choi-Sledeski YM, Becker MR, Ewing WR, Pauls HW, Spada AP, Mikol V. Molecular structures of human factor Xa complexed with ketopiperazine inhibitors: preference for a neutral group in the S1 pocket. J Med Chem. 2003; 46:685-90.

10. Gerotziafas TG, Samama MM. Heterogeneity of Synthetic Factor Xa Inhibitors. Curr Pharm Des. 2005; 11:3855-3876. 